Infinite Dimensional Analysis, Quantum Probability and Related Topics

Vol. 9, No. 2 (2006) 215-248

(C) World Scientific Publishing Company

\title{
A QUANTUM APPROACH TO LAPLACE OPERATORS
}

\author{
LUIGI ACCARDI \\ Centro Vito Volterra, Facultà di Economia, Università di Tor Vergata, \\ Via di Tor Vergata, 00133 Roma, Italy \\ accardi@volterra.mat.uniroma2.it \\ ABDESSATAR BARHOUMI \\ Nabeul Preparatory Engineering Institute, \\ Mrezgua University Compus, 8000 Nabeul, Tunisia \\ abdessatar.barhoumi@ipein.rnu.tn \\ HABIB OUERDIANE \\ Department of Mathematics, Faculty of Sciences of Tunis, \\ University of Tunis El Manar, 1060 Tunis, Tunisia \\ habib.ouerdiane@fst.rnu.tn
}

Received 2 March 2005

Communicated by H.-H. Kuo

\begin{abstract}
In this paper, a theory of stochastic processes generated by quantum extensions of Laplacians is developed. Representations of the associated heat semigroups are discussed by means of suitable time shifts. In particular the quantum Brownian motion associated to the Lévy-Laplacian is obtained as the usual Volterra-Gross Laplacian using the Cesàro Hilbert space as initial space of our process as well as multiplicity space of the associated white noise.
\end{abstract}

Keywords: Cesàro scalar product; Cesàro Hilbert space; distribution; Gel'fand triple; Lie algebra; Markovian semigroup; Wiener process; quantum shift; quantum heat flow; quantum Laplacian; quantum Lévy Brownian motion; white noise.

AMS Subject Classification: primary: 60J65, secondary: 60J45, 60H40

\section{Introduction}

The difference between the standard, classical, $\mathbb{R}^{d}$-valued Brownian motion and the associated increment process, the white noise, is just in the initial (i.e. time 0 ) random variable. In many cases one chooses the initial random variable to be concentrated at a point (e.g. the origin of $\mathbb{R}^{d}$ ) and one identifies the two spaces.

This identification, however, cannot be extended to the time shifts of the corresponding processes. In fact the time shift of the Brownian motion brings the initial 
random variable $W_{0}$ to the random variable at time $t, W_{t}$ (cf. Sec. 2.1) and this induces a shift in the increments.

On the contrary, the shift of the white noise only translates the increments but leaves the initial random variable (hence the functions of it) invariant (cf. Sec. 2.2).

A consequence of this fact is that the Markov semigroup, associated to the Brownian motion, is the heat semigroup while the one associated to the white noise, is the identity semigroup.

In the context of the Hudson-Parthasarathy theory, the white noise space is identified to a Fock space (cf. Secs. 2.2 and 2.3) and the classical white noise time shift is extended to an automorphism of the algebra of bounded operators on the Fock space.

In the Obverwolfach workshop on Quantum Probability in 1987, Meyer ${ }^{27}$ posed the problem of the existence of a quantum extension of the time shift of the Brownian motion (cf. Sec. 2.3) for a precise formulation).

A first solution to Meyer's problem was given by Accardi, ${ }^{1}$ during the Oberwolfach meeting and is based on the following idea. The classical Brownian motion time shift

$$
W_{0} \mapsto W_{t}=W_{0}+\left(W_{t}-W_{0}\right)
$$

can be obtained by adding the increment to the initial random variable.

Notice that, in order for the addition to make sense, the initial random variable $W_{0}$ must take values in the same space where the increments take values. In order to extend this obvious requirement to a more general context, one is led to consider a white noise whose multiplicity space coincides with the (complexification of the) Hilbert space where the initial distribution takes values (cf. the discussion at the end of Sec. 2.1).

The construction in Ref. 1, of a solution to Meyer's problem, considered only an $\mathbb{R}$-valued Brownian motion and was based on the remark that the increment process $W_{t}-W_{0}$ corresponds, in the Fock space, to the momentum field $P_{[0, t]}$ (cf. Sec. 3.1).

Identifying the initial random variable with the position operator $q_{0}$, one is led to the identification

$$
W_{t}=W_{0}+\left(W_{t}-W_{0}\right) \equiv q_{0} \otimes 1+1 \otimes P_{[0, t]},
$$

where the tensor product emphasizes that the two operators act on different spaces. Replacing the position operator $q_{0}$ by an arbitrary operator on the initial space, one obtains a quantum extension of the classical Brownian motion-time shift which, after exponentiation, leads to 1-parameter endomorphism group with the property that the associated Markov semigroup, when restricted to the position algebra, gives the usual heat semigroup.

Several problems were left open in Ref. 1 . In particular:

(i) The shift (1.1) is not fully quantum because the noise algebra is generated by $\left(P_{[0, t]}\right)$ and $\left(Q_{[0, t]}\right)$. 
(ii) Do there exist "quantum time shifts" which involve both position and momentum and still produce solutions to the Meyer's problem. Shifts with this property are constructed in Sec. 3.2, they are called "Lie algebra type shifts". The associated "Laplacians" are described in Sec. 4.1. A class of quantum diffusions, leading to these Laplacians, is described in Sec. 4.2. In Sec. 5 we discuss a quantum analogue of the "Euclidean invariance" of the classical Laplacian and we show that, if we take the full symplectic group as quantum analogue of the Euclidean group, then rotation invariant generators cannot be implemented by Lie algebra type shifts.

On the other hand, if we drop this requirement, i.e. if we allow "quantum time shifts" of a more general kind, then we can construct "quantum heat semigroups" which extend the usual Laplacian in a stronger sense than what was required by Meyer's problem, namely: their restriction to both position and momentum algebras give the classical Laplacian. Finally, in Sec. 6 we apply these ideas to the Lévy Laplacian and we prove that our "quantum extension" allows to unify the notion of Volterra-Gross and Lévy-Laplacians, and to prove that the difference between them can be reduced to a different choice of the "multiplicity space" of the white noise which, according to the previous discussion, is the complexification of the Hilbert space where the classical Brownian motion takes values.

\section{From Classical to Quantum Laplacians}

\subsection{The time shift of the Brownian motion}

Let $\left(W_{t}^{d}\right)_{t \geq 0}$ be the Wiener process on $\mathbb{R}^{d}$ with initial condition $W_{0}$, assumed to be independent of $\left(W_{t}^{d}\right)_{t \geq 0}$, and let $P_{0}$ denote the distribution of $W_{0}$. It is known that, if $f \in L_{\mathbb{C}}^{\infty}\left(\mathbb{R}^{d}\right)$ is a bounded (Borel) measurable complex valued function on $\mathbb{R}$, then for any $t \geq 0$

$$
\begin{aligned}
E\left(f\left(W_{t}^{d}\right)\right) & =E_{\{0\}}\left(E_{0]}\left(f\left(W_{t}^{d}\right)\right)\right)=\int_{\mathbb{R}^{d}} P_{0}\left(d x_{0}\right)\left(e^{t \Delta / 2} f\right)\left(W_{0}\right) \\
& =\int_{\mathbb{R}^{d}} P_{0}\left(d x_{0}\right) \int_{\mathbb{R}^{d}} d x \frac{e^{-\left|x-W_{0}\right|^{2} / 2 t}}{(\sqrt{2 \pi t})^{d}} f(x),
\end{aligned}
$$

where $E, E_{\{0\}}$ and $E_{0]}$ denote respectively the expectation, $\mathcal{F}_{\{0\}}$-conditional expectation and the $\mathcal{F}_{0]}$-conditional expectation with respect to the Wiener measure and $\Delta$ is the Laplacian on $L_{\mathbb{C}}^{2}\left(\mathbb{R}^{d}\right)$.

Denote $v_{t}^{\circ}$ the time shift for the Wiener process:

$$
v_{t}^{\circ}\left(F\left(W_{t_{1}}^{d}, \ldots, W_{t_{n}}^{d}\right)\right)=F\left(W_{t_{1}+t}^{d}, \ldots, W_{t_{n}+t}^{d}\right),
$$

where $F: \mathbb{R}^{n} \rightarrow \mathbb{R}$ is Borel measurable and denote $j_{t}$ the flow of the Wiener process:

$$
j_{t}: f \in L_{\mathbb{C}}^{\infty}\left(\mathbb{R}^{d}\right) \equiv L_{\mathbb{C}}^{\infty}\left(W_{0}\right) \rightarrow j_{t}(f):=f\left(W_{t}^{d}\right) \in L_{\mathbb{C}}^{\infty}\left(\mathbb{R}^{d} ; L_{\mathbb{C}}^{2}\left(W^{d}\right)\right),
$$


where $L_{\mathbb{C}}^{2}\left(W^{d}\right)$ denotes the $L^{2}$-space on the Wiener space. Then the identity $(2.1)$ can be rewritten as

$$
e^{-t L_{0}}(f)\left(W_{0}\right):=E_{0]}\left(v_{t}^{\circ}\left(j_{0}(f)\right)\right)=e^{t \Delta / 2} f\left(W_{0}\right) .
$$

In this sense we say that the generator of the semigroup associated to the Wiener process on $\mathbb{R}^{d}$ is the Laplacian.

Notice that, if $\mathcal{H}_{S}:=L_{\mathbb{C}}^{\infty}\left(\mathbb{R}^{d}\right) \equiv L_{\mathbb{C}}^{\infty}\left(W_{0}\right)$ is the space of possible initial data $\left(W_{0}\right)$, then the space of the Wiener process is

$$
\mathcal{H}_{S} \otimes L_{\mathbb{C}}^{2}\left(W^{d}\right) \equiv L_{\mathbb{C}}^{\infty}\left(\mathbb{R}^{d} ; L_{\mathbb{C}}^{2}\left(W^{d}\right)\right) .
$$

We say that the time shift $v_{t}^{\circ}$ can be unitarily implemented if there exists a 1parameter unitary group

$$
V_{t}^{\circ}: \mathcal{H}_{S} \otimes L_{\mathbb{C}}^{2}\left(W^{d}\right) \rightarrow \mathcal{H}_{S} \otimes L_{\mathbb{C}}^{2}\left(W^{d}\right)
$$

such that

$$
v_{t}^{\circ}(\cdot)=V_{-t}^{\circ}(\cdot) V_{t}^{\circ}: L_{\mathbb{C}}^{\infty}\left(\mathbb{R}^{d} ; L_{\mathbb{C}}^{2}\left(W^{d}\right)\right) \rightarrow L_{\mathbb{C}}^{\infty}\left(\mathbb{R}^{d} ; L_{\mathbb{C}}^{2}\left(W^{d}\right)\right),
$$

where $L_{\mathbb{C}}^{\infty}\left(\mathbb{R}^{d} ; L_{\mathbb{C}}^{2}\left(W^{d}\right)\right)$ is identified to an Abelian algebra of bounded operators acting by pointwise multiplication on $L_{\mathbb{C}}^{2}\left(\mathbb{R}^{d} ; L_{\mathbb{C}}^{2}\left(W^{d}\right)\right)$. In this case the time shift of the Wiener process can be extended to a 1-parameter (semi)-group of endomorphisms, still denoted $v_{t}^{\circ}$, of the algebra (of bounded operators)

$$
\mathcal{B}\left(\mathcal{H}_{S} \otimes L_{\mathbb{C}}^{2}\left(W^{d}\right)\right) \equiv \mathcal{B}\left(\mathcal{H}_{S}\right) \otimes \mathcal{B}\left(L_{\mathbb{C}}^{2}\left(W^{d}\right)\right)
$$

which respects the quantum time filtration, in the sense that:

$$
v_{t}^{\circ}\left(b_{0}\right) \in \mathcal{B}_{t]}:=\mathcal{B}\left(\mathcal{H}_{S}\right) \otimes \mathcal{B}\left(L_{[0, t]}^{2}\left(W^{d}\right)\right), \quad \forall b_{0} \in \mathcal{B}\left(\mathcal{H}_{S}\right) .
$$

The extension property means that, for any $f \in L_{\mathbb{C}}^{\infty}\left(\mathbb{R}^{d}\right)$

$$
v_{t}^{\circ}\left(M_{f}\right)=M_{f\left(W_{t}^{d}\right)},
$$

where $M_{f}$ denotes multiplication by the function $f$.

Now notice that

$$
L_{\mathbb{C}}^{2}\left(W^{d}\right) \equiv L_{\mathbb{C}}^{2}\left(W^{1}\right) \otimes \mathbb{C}^{d}
$$

and $\mathbb{C}^{d}$ can be considered as the complexification of the real Hilbert space $\mathbb{R}^{d}$. Furthermore,

$$
L_{\mathbb{C}}^{2}\left(\mathbb{R} ; \mathbb{R}^{d}\right) \equiv L_{\mathbb{C}}^{2}(\mathbb{R}) \otimes \mathbb{C}^{d} \equiv \Gamma\left(\mathbb{C}^{d}\right),
$$

where $\Gamma$ denotes the Fock space. Therefore, replacing $\mathbb{R}^{d}$ and $\mathbb{C}^{d}$ by a real Hilbert space $\mathcal{K}_{S, r}$ and its complexification $\mathcal{K}_{S}$, respectively, we can extend the above picture replacing $\mathbb{C}^{d}$ by an arbitrary complex Hilbert space $\mathcal{K}_{S}$ with a real structure

$$
\mathcal{K}_{S} \equiv \mathcal{K}_{S, r} \oplus i \mathcal{K}_{S, r}
$$


the initial $L^{2}$-space $L_{\mathbb{C}}^{2}\left(\mathbb{R}^{d}\right) \equiv \Gamma\left(\mathbb{C}^{d}\right)$ by the Fock space $\mathcal{H}_{S}=\Gamma\left(\mathcal{K}_{S}\right)$ over $\mathcal{K}_{S}$ and the noise space

$$
L_{\mathbb{C}}^{2}\left(W^{1}\right) \otimes \mathbb{C}^{d}=L^{2}\left(W^{1} ; \mathbb{C}^{d}\right)
$$

by $L^{2}\left(W^{1} ; \mathcal{K}_{S}\right) \equiv L_{\mathbb{C}}^{2}\left(W^{1}\right) \otimes \mathcal{K}_{S}$. This corresponds to replacing the $\mathbb{R}^{d}$-valued Brownian motion by a $\mathcal{K}_{S, r}$-valued Brownian motion. It is known that this extension allows to deal with the usual infinite dimensional Volterra Laplacian. We will prove that it includes also the Lévy-Laplacian.

From now on $W=W^{1}$ will denote the 1-dimensional ( $\mathbb{R}$-valued) classical Wiener process starting from the origin in $\mathbb{R}$. This is canonically identified to the space of the increments of the Wiener process in the sense that the filtration generated by $\left\{W_{t} ; 0 \leq t \leq \rho\right\}$ coincides with the filtration generated by $\left\{W_{t}-W_{s} ; 0 \leq s, t \leq \rho\right\}$. Here $\rho$ is an arbitrary fixed positive real number. We will see that this identification cannot be extended to the corresponding time shifts.

\subsection{The shift of white noise}

There is a known natural identification

$$
L_{\mathbb{C}}^{2}(W) \equiv \Gamma\left(L^{2}(\mathbb{R})\right)
$$

which induces the identification

$$
\mathcal{H}_{S} \otimes L_{\mathbb{C}}^{2}(W) \equiv \mathcal{H}_{S} \otimes \Gamma\left(L^{2}(\mathbb{R}) .\right.
$$

Moreover, one can choose this identification so that the operator of multiplication by $W_{t}$ goes into

$$
W_{t}=A_{t}^{+}+A_{t}
$$

where $\left(A_{t}\right)$ is the boson Fock-Brownian motion characterized by

$$
\begin{gathered}
{\left[A_{t}, A_{s}^{+}\right]=s \wedge t,} \\
A_{t} \Phi=0
\end{gathered}
$$

and $\Phi$ is the vacuum vector. Denote by $\theta_{t}$ the right shift on $L^{2}\left(\mathbb{R}_{+}, \mathcal{K}_{S}\right)$,

$$
\left(\theta_{t} f\right)(s)= \begin{cases}f(s-t), & 0 \leq t \leq s \\ 0 & 0 \leq s<t\end{cases}
$$

$\theta_{t}$ is isometric with left inverse $\theta_{t}^{*}(f)(s)=f(s+t)$. Notice that, for $f \in L^{2}\left(\mathbb{R}_{+}, \mathcal{K}_{S}\right)$, we have

$$
\theta_{t}^{*} M_{f} \theta_{t}=M_{f(\cdot+t)} .
$$

Let $\Gamma\left(\theta_{t}\right)$ be the second quantization of $\theta_{t}$ acting on $\Gamma\left(L^{2}\left(\mathbb{R}_{+}, \mathcal{K}_{S}\right)\right)$ and characterized by its action on exponential vectors $\psi(f) \in \Gamma\left(L^{2}\left(\mathbb{R}_{+}, \mathcal{K}_{S}\right)\right)$ by

$$
\Gamma\left(\theta_{t}\right) \psi(f)=\psi\left(\theta_{t} f\right) .
$$


According to the discussion in Sec. 1, the time shift $u_{t}^{\circ}$ is the 1-parameter endomorphisms semigroup of $\mathcal{B}\left(\mathcal{H}_{S}\right) \otimes \mathcal{B}\left(\Gamma\left(L^{2}\left(\mathbb{R}_{+}, \mathcal{K}_{S}\right)\right)\right)$ characterized by the property

$$
u_{t}^{\circ}\left(b_{0} \otimes b\right)=b_{0} \otimes \Gamma\left(\theta_{t}^{*}\right) b \Gamma\left(\theta_{t}\right), \quad b_{0} \in \mathcal{B}\left(\mathcal{H}_{S}\right), \quad b \in \mathcal{B}\left(\Gamma\left(L^{2}\left(\mathbb{R}_{+}, \mathcal{K}_{S}\right)\right)\right) .
$$

The shift $u_{t}^{\circ}$ is a normal ( $\sigma$-weakly continuous), left invertible, injective *endomorphism and satisfies the following:

(i) For $s, t \geq 0, u_{s}^{\circ} \circ u_{t}^{\circ}=u_{s+t}^{\circ}$;

(ii) For $s \geq 0$,

$$
u_{s}^{\circ}\left(\mathcal{B}\left(\mathcal{H}_{S}\right) \otimes \mathcal{B}\left(\Gamma\left(L^{2}\left(\mathbb{R}_{+}, \mathcal{K}_{S}\right)\right)\right)\right)=\mathcal{B}\left(\mathcal{H}_{S}\right) \otimes 1_{[0, s]} \otimes \mathcal{B}\left(\Gamma\left(L^{2}\left([s,+\infty), \mathcal{K}_{S}\right)\right)\right)
$$

where $1_{[0, s]}$ is the identity of $\mathcal{B}\left(\Gamma\left(L^{2}([0, s]), \mathcal{K}_{S}\right)\right)$.

\subsection{The vacuum conditional expectations}

In the identification (2.4), the conditional expectation $E_{0]}$ is mapped into the partial vacuum expectation value

$$
E_{0]}\left(b_{0} \otimes b\right)=\langle\Phi, b \Phi\rangle b_{0}, \quad b_{0} \in \mathcal{B}\left(\mathcal{H}_{S}\right), \quad b \in \mathcal{B}\left(\Gamma\left(L^{2}\left(\mathbb{R}_{+}\right) \otimes \mathcal{K}_{S}\right)\right) .
$$

More generally, the vacuum conditional expectations $\left\{E_{[s, t]} ; 0 \leq s \leq t \leq \infty\right\}$ onto the algebras $\mathcal{B}\left(\mathcal{H}_{S}\right) \otimes \mathcal{B}\left(\Gamma\left(L^{2}([s, t]) \otimes \mathcal{K}_{S}\right)\right)$ exist and are characterized by

$$
E_{[s, t]}\left(b_{0} \otimes b\right)=\left\langle\Phi, b_{[s, t]^{c}} \Phi\right\rangle b_{0} \otimes b_{[s, t]}, \quad b_{0} \in \mathcal{B}\left(\mathcal{H}_{S}\right), \quad b \in \mathcal{B}\left(\Gamma\left(L^{2}\left(\mathbb{R}_{+}\right) \otimes \mathcal{K}_{S}\right)\right),
$$

where $[s, t]^{c}=[0, s[\cup] t,+\infty[$ and

$$
b=b_{[0, s]} \otimes b_{[s, t]} \otimes b_{[t,+\infty[}
$$

is the decomposition of $b$ through the canonical factorization isomorphism

$$
\mathcal{B}:=\mathcal{B}\left(\Gamma\left(L^{2}\left(\mathbb{R}_{+}\right) \otimes \mathcal{K}_{S}\right)\right)=\mathcal{B}_{[0, s]} \otimes \mathcal{B}_{[s, t]} \otimes \mathcal{B}_{[t,+\infty[}
$$

with

$$
\begin{gathered}
\mathcal{B}_{[0, t]} \equiv \mathcal{B}_{t]}:=\mathcal{B}\left(\Gamma\left(L^{2}([0, t]) \otimes \mathcal{K}_{S}\right)\right) \otimes 1_{[t}, \\
\mathcal{B}_{[t,+\infty[} \equiv \mathcal{B}_{[t}:=1_{t]} \otimes \mathcal{B}\left(\Gamma\left(L^{2}([t,+\infty)) \otimes \mathcal{K}_{S}\right)\right)
\end{gathered}
$$

and more generally

$$
\mathcal{B}_{[s, t]}=1_{s]} \otimes \mathcal{B}\left(\Gamma\left(L^{2}([s, t]) \otimes \mathcal{K}_{S}\right)\right) \otimes 1_{[t} .
$$

As is easily understood, $1_{t]}, 1_{[t}$ and $1_{[s, t]}$ stand for the identity of $\mathcal{B}\left(\Gamma\left(L^{2}([0, t]) \otimes\right.\right.$ $\left.\left.\mathcal{K}_{S}\right)\right), \mathcal{B}\left(\Gamma\left(L^{2}([s, t]) \otimes \mathcal{K}_{S}\right)\right)$ and $\mathcal{B}\left(\Gamma\left(L^{2}([t, \infty)) \otimes \mathcal{K}_{S}\right)\right)$, respectively.

The $E_{[s, t]}$ 's satisfy the condition:

$$
E_{[s, t]} \circ E_{\left[s^{\prime}, t^{\prime}\right]}=E_{[s, t]}, \quad \text { if }[s, t] \subseteq\left[s^{\prime}, t^{\prime}\right] .
$$

We shall write $E_{t]}$ for $E_{[0, t]}$. 
It is easily checked that, for any $s, t \geq 0$, the following identity holds

$$
u_{t}^{\circ} \circ E_{s]}=E_{s+t]} \circ u_{t}^{\circ} \text {. }
$$

Moreover, the usual properties of conditional expectations hold, for example

$$
E_{0]}\left(a_{1} b a_{2}\right)=E_{0]}\left(a_{1} E_{t]}(b) a_{2}\right)
$$

for arbitrary $a_{1}, a_{2}, b \in \mathcal{B}$ and $t \geq 0$. From this and the preceding discussion (see Sec. 2.1), it follows that, for any $b_{0} \in \mathcal{B}\left(\mathcal{H}_{S}\right)$, one has

$$
u_{t}^{\circ}\left(b_{0}\right)=b_{0} \equiv b_{0} \otimes 1_{\Gamma\left(L^{2}(\mathbb{R})\right)}=: j_{0}\left(b_{0}\right)
$$

and therefore the associated Markov semigroup is also trivial:

$$
E_{0]}\left(u_{t}^{\circ}\left(b_{0}\right)\right)=b_{0} \text {. }
$$

\subsection{Meyer's problem}

From (2.8) it follows that, in the identification (2.4), the time shift of the Wiener space is not identified with the time shift in the corresponding Fock space because the former gives rise to the identity semigroup while the latter to the usual heat semigroup. This remark motivated Meyer to pose the problem of the existence of a "time shift", i.e. a 1-parameter endomorphism semigroup $v_{t}^{\circ}$ of

$$
\mathcal{B}\left(\mathcal{H}_{S}\right) \otimes \mathcal{B}\left(\Gamma\left(L^{2}(\mathbb{R})\right)\right)
$$

with the property that for any $f \in L_{\mathbb{C}}^{\infty}(\mathbb{R})$

$$
P^{t}\left(M_{f}\right)=E_{0]}\left(v_{t}^{\circ}\left(j_{0}\left(M_{f}\right)\right)\right)=M_{e^{t \Delta / 2} f} .
$$

Suppose that the answer to Meyer's question is positive and notice that, since by assumption $j_{0}$ is defined on $\mathcal{B}\left(\mathcal{H}_{S}\right)$ and $v_{t}^{\circ}$ is defined on the whole algebra (2.10), the left-hand side of $(2.11)$ can be extended to the whole of $\mathcal{B}\left(\mathcal{H}_{S}\right)$. In other words, the expression

$$
E_{0]}\left(v_{t}^{\circ}\left(j_{0}\left(b_{0}\right)\right)\right)=: P^{t}\left(b_{0}\right)
$$

makes sense for each $b_{0} \in \mathcal{B}\left(\mathcal{H}_{S}\right)$. If $P^{t}$ is a Markov semigroup, then it has a generator $L$ :

$$
E_{0]}\left(v_{t}^{\circ}\left(j_{0}\left(b_{0}\right)\right)\right)=P^{t}\left(b_{0}\right)=e^{-t L}\left(b_{0}\right) ; \quad \forall b_{0} \in \mathcal{B}\left(\mathcal{H}_{S}\right) .
$$

Because of (2.11) the generator $L$ has the property that

$$
L\left(M_{f}\right)=M_{\left(-\frac{1}{2} \Delta f\right)},
$$

i.e. the restriction of $L$ to the multiplication operators coincides with the classical Laplacian. Therefore, we come to a first path to a quantum extension of the Laplace operator.

Definition 2.1. A quantum Markov generator $L$ on $\mathcal{B}\left(L^{2}(\mathbb{R})\right)$ is called a quantum extension of the classical Laplacian on $L^{2}(\mathbb{R})$ if the multiplication by twice differentiable bounded functions are in the domain of $L$ and on such functions (2.12) holds. 


\section{The Quantum Heat Flow of the CCR Algebra}

\subsection{The Heisenberg Lie algebra}

Let $\mathcal{H}_{1}$ be a complex Hilbert space with a real structure, this means that there exists a real Hilbert subspace $\mathcal{H}_{1, r}$ of $\mathcal{H}_{1}$ with real-valued scalar product characterized by the condition

$$
\langle f, i g\rangle_{\mathcal{H}_{1}}=i\langle f, g\rangle_{\mathcal{H}_{1, r}}, \quad f, g \in \mathcal{H}_{1, r}
$$

and an identification:

$$
\mathcal{H}_{1} \equiv \mathcal{H}_{1, r} \dot{+} i \mathcal{H}_{1, r}
$$

where the symbol $\dot{+}$ denotes that the Hilbert space sum is direct, but not orthogonal (cf. (3.1)). This means that, in the identification (3.2), each $z \in \mathcal{H}_{1}$ has a unique decomposition

$$
z=a+i b, \quad a, b \in \mathcal{H}_{1, r}
$$

and the conjugation map

$$
z \mapsto z^{*}:=\bar{z}=a-i b
$$

is well defined. For example in the 1-dimensional case $\mathcal{H}_{1}=\mathbb{C}$ and $z^{*}=\bar{z}$ is the usual complex conjugation.

In the identification $(3.2)$ the scalar product $\langle.,.\rangle \equiv\langle.,\rangle_{\mathcal{H}_{1}}$ on $\mathcal{H}_{1}$ is uniquely determined by its restriction $\langle., .\rangle_{r}$ on $\mathcal{H}_{1, r}$ through the algebraic identities:

$$
\begin{aligned}
& \operatorname{Im}\left\langle f+i g, f^{\prime}+i g^{\prime}\right\rangle=\left\langle f, g^{\prime}\right\rangle_{r}-\left\langle g, f^{\prime}\right\rangle_{r}, \\
& \operatorname{Re}\left\langle f+i g, f^{\prime}+i g^{\prime}\right\rangle=\operatorname{Im}\left\langle f+i g,-g^{\prime}+i f^{\prime}\right\rangle=\left\langle f, f^{\prime}\right\rangle_{r}+\left\langle g, g^{\prime}\right\rangle_{r} .
\end{aligned}
$$

Definition 3.1. The complex Heisenberg Lie algebra over $\mathcal{H}_{1}$ is the complex $*$-Lie algebra with generators $q_{0}(z), p_{0}(z), z \in \mathcal{H}_{1}$ and central element $E_{0}$, i.e.

$$
\mathcal{L}_{S}:=\operatorname{Lie}\left\{q_{0}(z), p_{0}(z), E_{0} ; z \in \mathcal{H}_{1}\right\}
$$

and relations

$$
\begin{aligned}
p_{0}(z)^{*} & =p_{0}\left(z^{*}\right) ; \quad q_{0}(z)^{*}=q_{0}\left(z^{*}\right) ; \quad E_{0}^{*}=-E_{0} ; \quad q_{0}(i z)=p_{0}(z), \quad z \in \mathcal{H}_{1} \\
{\left[X, E_{0}\right] } & =0, \quad \forall X \in \mathcal{L}_{S} \\
{\left[p_{0}(z), q_{0}\left(z^{\prime}\right)\right] } & =-i \operatorname{Re}\left\langle z, z^{\prime}\right\rangle E_{0} ; \quad \forall z, z^{\prime} \in \mathcal{H}_{1} \\
z & \mapsto p_{0}(z), z \mapsto q_{0}(z) \quad \text { are real linear maps. }
\end{aligned}
$$

We will denote $\mathcal{L}_{S, r}$ the Lie algebra obtained replacing $\mathcal{H}_{1}$ by $\mathcal{H}_{1, r}$ in (3.5).

Definition 3.2. A representation of $\mathcal{L}_{S}$ is a triple $\{\mathcal{H}, \mathcal{D}, \pi\}$ where $\mathcal{H}$ is a Hilbert space, $\mathcal{D} \subseteq \mathcal{H}$ is a dense subspace and $\pi$ is a $*$-homomorphism of $\mathcal{L}_{S}$ onto a Lie algebra of linear operators on $\mathcal{H}$ having $\mathcal{D}$ as a common invariant domain. 
We will discuss various representations of $\mathcal{L}_{S}$, acting on the boson Fock space $\mathcal{H}:=\Gamma\left(\mathcal{H}_{1}\right)$, over one of the following three spaces:

$$
\begin{aligned}
& \mathcal{H}_{1}=\mathcal{K}_{S} \\
& \mathcal{H}_{1}=L^{2}\left(\mathbb{R} ; \mathcal{K}_{S}\right) \equiv L^{2}(\mathbb{R}) \otimes \mathcal{K}_{S}, \\
& \mathcal{H}_{1}=\mathcal{K}_{S} \oplus L^{2}\left(\mathbb{R} ; \mathcal{K}_{S}\right)
\end{aligned}
$$

Here $\mathcal{K}_{S}$ is a complex Hilbert space as in the previous section. For the definition of the basic operators acting on the Fock space, we refer to Parthasarathy's monograph. ${ }^{31}$ We only recall that the creator map

$$
F \in \mathcal{H}_{1} \mapsto A^{+}(F)
$$

is complex linear and the CCR hold

$$
\left[A(F), A^{+}\left(F^{\prime}\right)\right]=\left\langle F, F^{\prime}\right\rangle 1 ; \quad F, F^{\prime} \in \mathcal{H}_{1},
$$

where 1 is the identity of $\mathcal{B}\left(\Gamma\left(\mathcal{H}_{1}\right)\right)$. The Weyl operators are defined by

$$
F \in \mathcal{H}_{1} \mapsto W_{0}(F):=e^{i\left(A^{+}(F)+A(F)\right)} .
$$

They are unitary and the norm closure, in $\Gamma\left(\mathcal{H}_{1}\right)$, of the algebra generated by them, denoted $W\left(\mathcal{H}_{1}\right)$, will be called the Weyl algebra over $\mathcal{H}_{1}$. For each pair $F, G \in \mathcal{H}_{1}$ the CCR in bounded form hold:

$$
W(F) W(G)=e^{-i \operatorname{Im}\langle F, G\rangle} W(F+G)
$$

and $\left\{W(F) ; F \in \mathcal{H}_{1}\right\}$ is irreducible on $\Gamma\left(\mathcal{H}_{1}\right)$. The position (resp. momentum) field operators are defined, for $F \in \mathcal{H}_{1}$, by:

$$
\begin{gathered}
q(F):=\frac{1}{\sqrt{2}}\left(A(F)+A^{+}(F)\right), \\
p(F):=\frac{1}{i \sqrt{2}}\left(A(F)-A^{+}(F)\right)=q(i F),
\end{gathered}
$$

or equivalently

$$
A(F)=\frac{1}{\sqrt{2}}(q(F)+i p(F)) ; \quad A^{+}(F)=\frac{1}{\sqrt{2}}(q(F)-i p(F)) .
$$

In particular, if $W(F)$ denote the unitary (Weyl) operator at $F \in \mathcal{H}_{1}$

$$
W(F):=e^{i\left(A(F)+A^{+}(F)\right)}=e^{i \sqrt{2} q(F)} ; \quad W(i F)=e^{i \sqrt{2} p(F)} .
$$

For each $F, G \in \mathcal{H}_{1}, q(F), p(F)$ are essentially self-adjoint and satisfy the CCR

$$
\begin{gathered}
{[q(F), q(G)]=[p(F), p(G)]=i \operatorname{Im}\langle F, G\rangle 1,} \\
{[p(F), q(G)]=-i \operatorname{Re}\langle F, G\rangle 1 .}
\end{gathered}
$$

The $\mathcal{K}_{S, r}$-valued (boson Fock) white noise space is the Fock space

$$
\Gamma\left(L^{2}\left(\mathbb{R} ; \mathcal{K}_{S}\right)\right) \equiv \Gamma\left(L^{2}(\mathbb{R}) \otimes \mathcal{K}_{S}\right) .
$$


For $t \in \mathbb{R}_{+}$we denote

$$
A_{t}^{+}: z \in \mathcal{K}_{S} \mapsto A^{+}\left(\chi_{[0, t]} \otimes z\right)=: A_{t}^{+}(z)
$$

which is a complex linear map. Similarly we define, for $t \geq 0$ and $z \in \mathcal{K}_{S}$, the operators

$$
q_{t}(z):=q\left(\chi_{[0, t]} \otimes z\right) ; \quad p_{t}(z):=p\left(\chi_{[0, t]} \otimes z\right)
$$

which are real linear maps in $z$.

We fix the initial space (space of "initial values") to be

$$
\mathcal{H}_{S}:=\Gamma\left(\mathcal{K}_{S}\right)
$$

and the noise space to be (cf. the discussion at the end of Sec. 2.2)

$$
\Gamma\left(L^{2}\left(\mathbb{R}_{+}, \mathcal{K}_{S}\right)\right) \equiv \Gamma\left(L^{2}\left(\mathbb{R}_{+}\right) \otimes \mathcal{K}_{S}\right) .
$$

With these choices the space of this process is

$$
\mathcal{H}_{S} \otimes \Gamma\left(L^{2}\left(\mathbb{R}_{+} ; \mathcal{K}_{S}\right)\right)=\Gamma\left(\mathcal{K}_{S}\right) \otimes \Gamma\left(L^{2}\left(\mathbb{R}_{+} ; \mathcal{K}_{S}\right)\right)=\Gamma\left(\mathcal{K}_{S} \oplus L^{2}\left(\mathbb{R}_{+} ; \mathcal{K}_{S}\right)\right) .
$$

For the constructions we are going to do, it is necessary that the "multiplicity" (or "polarization") space of the noise coincides with the initial space.

From the above considerations, we see that any real structure on $\mathcal{K}_{S}$ uniquely induces a real structure on $L^{2}\left(\mathbb{R}_{+} ; \mathcal{K}_{S}\right)$ given by

$$
L^{2}\left(\mathbb{R}_{+} ; \mathcal{K}_{S}\right)=L^{2}\left(\mathbb{R}_{+} ; \mathcal{K}_{S, r}\right) \dot{+} i L^{2}\left(\mathbb{R}_{+} ; \mathcal{K}_{S, r}\right),
$$

where $L^{2}\left(\mathbb{R}_{+} ; \mathcal{K}_{S, r}\right)$ is the space of $\mathcal{K}_{S, r^{-}}$valued functions on $\mathbb{R}_{+}$with scalar product

$$
\langle F, G\rangle_{r}:=\int_{\mathbb{R}_{+}}\langle F(t), G(t)\rangle_{\mathcal{K}_{S, r}} d t .
$$

\subsection{A class of Markov flows}

In this section we define a family of representations $\hat{j}_{t}$ of $\mathcal{L}_{S}$, acting on the Fock space over the space (3.7), through the identities $\left(z \in \mathcal{K}_{S}, t \geq 0\right)$

$$
\begin{gathered}
\hat{j}_{t}\left(p_{0}(z)\right):=p_{0}(z) \otimes 1+1_{0} \otimes Y_{[0, t]}\left(T_{1} z\right) \equiv p_{0}(z)+Y_{[0, t]}\left(T_{1} z\right), \\
\hat{j}_{t}\left(q_{0}(z)\right):=q_{0}(z) \otimes 1+1_{0} \otimes X_{[0, t]}\left(T_{2} z\right) \equiv q_{0}(z)+X_{[0, t]}\left(T_{2} z\right), \\
\hat{j}_{t}\left(E_{0}\right):=E_{0}+Z_{[0, t]},
\end{gathered}
$$

where $T_{1}$ and $T_{2}$ are real linear operators on $\mathcal{K}_{S}$ and $X_{[0, t]}, Y_{[0, t]}, Z_{[0, t]}(t \geq 0)$ are the real linear maps:

$$
\begin{aligned}
z \in \mathcal{K}_{S} \mapsto X_{[0, t]}(z) & =X\left(\chi_{[0, t]} \otimes z\right):=\alpha q_{t}(z)+\beta p_{t}(z) \\
& =\frac{u}{\sqrt{2}} A_{t}^{+}(z)+\frac{\bar{u}}{\sqrt{2}} A_{t}(z)
\end{aligned}
$$




$$
\begin{aligned}
& z \in \mathcal{K}_{S} \mapsto Y_{[0, t]}(z)=Y\left(\chi_{[0, t]} \otimes z\right):=\gamma q_{t}(z)+\delta p_{t}(z) \\
&=\frac{v}{\sqrt{2}} A_{t}^{+}(z)+\frac{\bar{v}}{\sqrt{2}} A_{t}(z), \\
& Z_{[0, t]} \hat{\in} \mathcal{B}\left(\Gamma\left(L^{2}\left(\mathbb{R}_{+}\right) \otimes \mathcal{K}_{S}\right)\right)
\end{aligned}
$$

where the symbol " $\hat{\in}$ " means that the operator is not necessarily bounded, $\alpha, \beta, \gamma$, $\delta \in \mathbb{R}$ and $u:=\alpha+i \beta, v:=\gamma+i \delta$ are non-zero complex numbers.

Since we want the $\hat{j}_{t}$ to be $*$-maps, the processes $X_{[0, t]}(z), Y_{[0, t]}(z)$ must be self-adjoint:

$$
X_{[0, t]}=X_{[0, t]}^{*} ; \quad Y_{[0, t]}=Y_{[0, t]}^{*}
$$

and $Z_{[0, t]}$ satisfies $Z_{[0, t]}=-Z_{[0, t]}^{*}$. The processes $X, Y$ and $Z$ are assumed to be additive, i.e.

$$
X_{[s, t]}+X_{[t, r]}=X_{[s, r]} ; \quad Y_{[s, t]}+Y_{[t, r]}=Y_{[s, r]} ; \quad Z_{[s, t]}+Z_{[t, r]}=Z_{[s, r]} .
$$

Moreover, if $(s, t) \cap(u, r)=\emptyset$, they satisfy

$$
\left[X_{[s, t]}, X_{[u, r]}\right]=\left[Y_{[s, t]}, Y_{[u, r]}\right]=\left[Z_{[s, t]}, Z_{[u, r]}\right]=0 .
$$

The maps $\hat{j}_{t}$ are then extended to $\mathcal{L}_{S}$ by complex linearity.

Lemma 3.1. In the above notations, for any $t \in \mathbb{R}_{+}$and $u, v \in \mathbb{C}$, the map $\hat{j}_{t}$ given by (3.18)-(3.20) is well defined if and only if

$$
v T_{1}(z)=u T_{2}(i z) ; \quad \forall z \in \mathcal{K}_{S} .
$$

In particular, $\hat{j}_{t}$ differs from the trivial embedding only if both $u, v \neq 0$ and there exists a nonzero real linear operator $T: \mathcal{K}_{S} \rightarrow \mathcal{K}_{S}$ such that $\hat{j}_{t}$ takes the form $\left(z \in \mathcal{K}_{S}, t \geq 0\right)$ :

$$
\begin{gathered}
\hat{j}_{t}\left(p_{0}(z)\right)=p_{0}(z)+\frac{1}{\sqrt{2}}\left[A_{t}^{+}(T z)+A_{t}(T z)\right], \\
\hat{j}_{t}\left(q_{0}(z)\right)=q_{0}(z)-\frac{1}{\sqrt{2}}\left[A_{t}^{+}(T(i z))+A_{t}(T(i z))\right], \\
\hat{j}_{t}\left(E_{0}\right)=E_{0}+Z_{[0, t]} .
\end{gathered}
$$

Proof. By definition, for any $z \in \mathcal{K}_{S}$

$$
\begin{aligned}
& \hat{j}_{t}\left(p_{0}(z)\right)=p_{0}(z)+Y_{[0, t]}\left(T_{1} z\right), \\
& \hat{j}_{t}\left(q_{0}(z)\right)=q_{0}(z)+X_{[0, t]}\left(T_{2} z\right) .
\end{aligned}
$$

Since the position and momentum field operators are related by

$$
p_{0}(z)=q_{0}(i z) ; \quad \forall z \in \mathcal{K}_{S}
$$

$\hat{j_{t}}$ must satisfy

$$
\hat{j}_{t}\left(p_{0}(z)\right)=\hat{j}_{t}\left(q_{0}(i z)\right) ; \quad \forall z \in \mathcal{K}_{S}
$$


or equivalently

$$
Y_{[0, t]}\left(T_{1} z\right)=X_{[0, t]}\left(T_{2}(i z)\right) ; \quad \forall z \in \mathcal{K}_{S}
$$

which is equivalent to

$$
u A_{t}^{+}\left(T_{2}(i z)\right)+\bar{u} A_{t}\left(T_{2}(i z)\right)=v A_{t}^{+}\left(T_{1}(z)\right)+\bar{v} A_{t}\left(T_{1}(z)\right) ; \quad \forall z \in \mathcal{K}_{S}
$$

equivalently

$$
\left(A_{t}^{+}+A_{t}\right)\left(u T_{2}(i z)-v T_{1}(z)\right)=0 ; \quad \forall z \in \mathcal{K}_{S} .
$$

This implies (3.23) because: $\left(A_{t}^{+}+A_{t}\right)(z)=0 \Leftrightarrow z=0$.

We see that the solutions of Eq. (3.23) define three nontrivial classes of parameters:

(i) $v=0 ; T_{2}=0$;

(ii) $u=0 T_{1}=0$;

(iii) $u \neq 0, v \neq 0$ and $T_{1}, T_{2}$ nonzero real linear operators such that (3.23) holds.

In cases (i) and (ii), we have respectively:

$$
\hat{j}_{t}\left(p_{0}(z)\right)=p_{0}(z) ; \quad \hat{j}_{t}\left(q_{0}(z)\right)=q_{0}(z) ; \quad \forall z \in \mathcal{K}_{S}
$$

and $\hat{j}_{t}$ is a trivial embedding. If $u \neq 0$ and $v \neq 0$, then

$$
\begin{aligned}
X_{[0, t]}\left(T_{2} z\right) & =\frac{u}{\sqrt{2}} A_{t}^{+}\left(T_{2} z\right)+\frac{\bar{u}}{\sqrt{2}} A_{t}\left(T_{2} z\right)=\frac{1}{\sqrt{2}} A_{t}^{+}\left(u T_{2} z\right)+\frac{1}{\sqrt{2}} A_{t}\left(u T_{2} z\right) \\
& =-\frac{1}{\sqrt{2}} A_{t}^{+}\left(v T_{1}(i z)\right)-\frac{1}{\sqrt{2}} A_{t}\left(v T_{1}(i z)\right) .
\end{aligned}
$$

Thus, denoting $T=v T_{1}$ one has

$$
\begin{aligned}
& X_{[0, t]}\left(T_{2} z\right)=-\frac{1}{\sqrt{2}}\left[A_{t}^{+}(T(i z))+A_{t}(T(i z))\right], \\
& Y_{[0, t]}\left(T_{1} z\right)=\frac{1}{\sqrt{2}}\left[A_{t}^{+}(T z)+A_{t}(T z)\right]
\end{aligned}
$$

and this completes the proof.

Proposition 3.1. In the notations of Lemma 3.1 with $u \neq 0, v \neq 0$, for any $t \geq 0$, the map $\hat{j}_{t}$ defined by (3.24)-(3.26) is a homomorphism of $*$-Lie algebras if and only if

$$
\operatorname{Im}\left\langle z, z^{\prime}\right\rangle Z_{[0, t]}=t \operatorname{Im}\left\langle T z, T z^{\prime}\right\rangle 1 ; \quad \forall z, z^{\prime} \in \mathcal{K}_{S}
$$

Proof. For any $z \in \mathcal{K}_{S}$ we have $\left(A_{t}^{+}(z)\right)^{*}=A_{t}(z)$. This implies

$$
\hat{j}_{t}\left(E_{0}^{*}\right)=\hat{j}_{t}\left(-E_{0}\right)=-\hat{j}_{t}\left(E_{0}\right)=-E_{0}-Z_{[0, t]}=\left(\hat{j}_{t}\left(E_{0}\right)\right)^{*}
$$

and therefore, the $*$-property of $\hat{j}_{t}$ is satisfied. 
The commutation relations give, for any $z, z^{\prime} \in \mathcal{K}_{S}$

$$
\begin{aligned}
& {\left[\hat{j}_{t}\left(p_{0}(z)\right), \hat{j_{t}}\left(q_{0}\left(z^{\prime}\right)\right)\right]} \\
& \quad=\left[p_{0}(z), q_{0}\left(z^{\prime}\right)\right]-\frac{1}{2}\left[A_{t}^{+}(T z)+A_{t}(T z), A_{t}^{+}\left(T\left(i z^{\prime}\right)\right)+A_{t}\left(T\left(i z^{\prime}\right)\right)\right] \\
& \quad=-i \operatorname{Re}\left\langle z, z^{\prime}\right\rangle E_{0}-\frac{1}{2}\left(\left[A_{t}^{+}(T z), A_{t}\left(T\left(i z^{\prime}\right)\right)\right]+\left[A_{t}(T z), A_{t}^{+}\left(T\left(i z^{\prime}\right)\right)\right]\right) \\
& \quad=-i \operatorname{Re}\left\langle z, z^{\prime}\right\rangle E_{0}-\frac{1}{2} t\left(-\left\langle T\left(i z^{\prime}\right), T z\right\rangle 1+\left\langle T z, T\left(i z^{\prime}\right)\right\rangle 1\right) \\
& \quad=-i \operatorname{Re}\left\langle z, z^{\prime}\right\rangle E_{0}-i t \operatorname{Im}\left\langle T z, T\left(i z^{\prime}\right)\right\rangle 1 .
\end{aligned}
$$

On the other hand

$$
\begin{aligned}
\hat{j}_{t}\left(\left[p_{0}(z), q_{0}\left(z^{\prime}\right)\right]\right) & =\hat{j}_{t}\left(-i \operatorname{Re}\left\langle z, z^{\prime}\right\rangle E_{0}\right) \\
& =-i \operatorname{Re}\left\langle z, z^{\prime}\right\rangle \hat{j}_{t}\left(E_{0}\right)=-i \operatorname{Re}\left\langle z, z^{\prime}\right\rangle E_{0}-i \operatorname{Re}\left\langle z, z^{\prime}\right\rangle Z_{[0, t]} .
\end{aligned}
$$

Therefore the commutation relations are preserved if and only if

$$
\operatorname{Re}\left\langle z, z^{\prime}\right\rangle Z_{[0, t]}=t \operatorname{Im}\left\langle T z, T\left(i z^{\prime}\right)\right\rangle 1 ; \quad \forall z, z^{\prime} \in \mathcal{K}_{S} .
$$

Replacing $z^{\prime}$ by $i z^{\prime}$ and using the fact that $\operatorname{Re}(i \omega)=-\operatorname{Im} \omega,(\omega \in \mathbb{C})$, this becomes $(3.27)$.

Lemma 3.2. Under condition (3.27), there exists $c \in \mathbb{R}$ (independent of $t$ ) such that

$$
\begin{aligned}
c \operatorname{Im}\left\langle z, z^{\prime}\right\rangle & =\operatorname{Im}\left\langle T z, T z^{\prime}\right\rangle ; \quad \forall z, z^{\prime} \in \mathcal{K}_{S} \\
Z_{[0, t]} & =c t ; \quad \forall t>0 .
\end{aligned}
$$

Proof. We distinguish two cases:

$$
\begin{aligned}
\operatorname{Im}\left\langle T z, T z^{\prime}\right\rangle=0 ; \quad & \forall z, z^{\prime} \in \mathcal{K}_{S} \\
\operatorname{Im}\left\langle T z_{1}, T z_{2}\right\rangle \neq 0 ; & \text { for some } z_{1}, z_{2} \in \mathcal{K}_{S} .
\end{aligned}
$$

In the first case one must have $Z_{[0, t]}=0$. In the second case one must also have $\operatorname{Im}\left\langle z_{1}, z_{2}\right\rangle \neq 0$ for the same pair $z_{1}, z_{2} \in \mathcal{K}_{S}$ and (3.27) implies that

$$
Z_{[0, t]}=t \frac{\operatorname{Im}\left\langle T z_{1}, T z_{2}\right\rangle}{\operatorname{Im}\left\langle z_{1}, z_{2}\right\rangle} .
$$

Therefore the quotient

$$
\frac{\operatorname{Im}\left\langle T z_{1}, T z_{2}\right\rangle}{\operatorname{Im}\left\langle z_{1}, z_{2}\right\rangle}=: c
$$

is a real number independent of $z_{1}, z_{2}$. In both cases the identity (3.28) holds. 
Definition 3.3. Let $\sigma: \mathcal{K}_{S} \times \mathcal{K}_{S} \rightarrow \mathbb{R}$ be a symplectic form, i.e. a bilinear form such that

$$
\sigma\left(z, z^{\prime}\right)=-\sigma\left(z^{\prime}, z\right) ; \quad \forall z, z^{\prime} \in \mathcal{K}_{S}
$$

A real linear map $T: \mathcal{K}_{S} \rightarrow \mathcal{K}_{S}$ is called symplectic if

$$
\sigma\left(T z, T z^{\prime}\right)=\sigma\left(z, z^{\prime}\right) ; \quad \forall z, z^{\prime} \in \mathcal{K}_{S} .
$$

$T$ is called anti-symplectic if

$$
\sigma\left(T z, T z^{\prime}\right)=-\sigma\left(z, z^{\prime}\right)=\sigma\left(z^{\prime}, z\right) ; \quad \forall z, z^{\prime} \in \mathcal{K}_{S} .
$$

Proposition 3.2. Let $T: \mathcal{K}_{S} \rightarrow \mathcal{K}_{S}$ be a real linear map and let $c \in \mathbb{R}$ denotes the constant given by (3.29). If $c \neq 0$, then for any $t>0, \hat{j}_{t}$ is a $*$-homomorphism of $*$-Lie algebras if and only if there exists $\omega \in \mathbb{C}$ such that $\omega T$ is either a symplectic or an anti-symplectic map with respect to $\sigma(.,)=.\operatorname{Im}\langle.,$.$\rangle .$

Proof. Using (3.29), condition (3.27) becomes

$$
c \operatorname{Im}\left\langle z, z^{\prime}\right\rangle=\operatorname{Im}\left\langle T z, T z^{\prime}\right\rangle ; \quad \forall z, z^{\prime} \in \mathcal{K}_{S} .
$$

Then we have the following two cases:

- If $c>0$ there exist $\omega \in \mathbb{C}$ such that

$$
c=\left|\frac{1}{\omega}\right|^{2} .
$$

In this case

$$
\operatorname{Im}\left\langle z, z^{\prime}\right\rangle=\operatorname{Im}\left\langle\omega T z, \omega T z^{\prime}\right\rangle ; \quad \forall z, z^{\prime} \in \mathcal{K}_{S}
$$

which proves that $\omega T$ is a symplectic map with respect to $\sigma\left(z, z^{\prime}\right)=\operatorname{Im}\left\langle z, z^{\prime}\right\rangle$.

- If $c<0$ there exist $\omega \in \mathbb{C}$ such that

$$
c=-\left|\frac{1}{\omega}\right|^{2}
$$

then

$$
\operatorname{Im}\left\langle z, z^{\prime}\right\rangle=-\operatorname{Im}\left\langle\omega T z, \omega T z^{\prime}\right\rangle ; \quad \forall z, z^{\prime} \in \mathcal{K}_{S} .
$$

This means that $\omega T$ is anti-symplectic map on $\mathcal{K}_{S}$ with respect to $\sigma\left(z, z^{\prime}\right)=$ $\operatorname{Im}\left\langle z, z^{\prime}\right\rangle$.

Remark 3.1. From the above discussion, we see that $\hat{j}_{t}\left(E_{0}\right)$ must have the form

$$
\hat{j}_{t}\left(E_{0}\right)=E_{0} \otimes 1+1_{0} \otimes c t 1 \equiv E_{0}+c t 1 ; \quad c \in \mathbb{R} .
$$

From now on, in this paper, $\left\{\hat{j}_{t} ; t \geq 0\right\}$ will be a family of $*$-homomorphisms of $*$-Lie algebras specified by the conditions (3.24), (3.25), (3.30). 
Theorem 3.1. For any $t \geq 0$, there exists a unique injective linear map $j_{t}^{\circ}$, defined on the algebraic linear span of the Weyl operators in the Weyl algebra $W_{0}\left(\mathcal{K}_{S}\right)$ over $\mathcal{K}_{S}$, and characterized by the identity

$$
j_{t}^{\circ}\left(e^{i X}\right):=e^{i \hat{j}_{t}(X)} ; \quad X \in \mathcal{L}_{S} .
$$

On the Weyl operator $W_{0}(z)=e^{i\left(a_{0}(z)+a_{0}^{+}(z)\right)},\left(z \in \mathcal{K}_{S}\right), j_{t}^{\circ}$ is given by:

$$
j_{t}^{\circ}\left(W_{0}(z)\right)=W_{0}(z) \otimes W\left(-\chi_{[0, t]} \otimes T(i z)\right) .
$$

Moreover, $j_{t}^{\circ}$ extends to a $*$-homomorphism of $C^{*}$-algebras (still denoted $j_{t}^{\circ}$ )

$$
j_{t}^{\circ}:=\exp \left(\hat{j_{t}}\right): W_{0}\left(\mathcal{K}_{S}\right) \rightarrow W_{0}\left(\mathcal{K}_{S}\right) \otimes W\left(L^{2}\left(\mathbb{R}_{+}\right) \otimes \mathcal{K}_{S}\right)
$$

if and only if

$$
\operatorname{Im}\left\langle T z, T z^{\prime}\right\rangle=0 ; \quad \forall z, z^{\prime} \in \mathcal{K}_{S} .
$$

Under this condition $j_{t}^{\circ}$ is a *-isomorphism onto its image and we have only one choice for $\hat{j}_{t}\left(E_{0}\right)$, namely:

$$
\hat{j_{t}}\left(E_{0}\right)=E_{0} \otimes 1=: \mathbf{1}
$$

Remark 3.2. Here and in all the remainder of this paper, $a_{0}(z), a_{0}^{+}(z)$ denote the annihilation and creation operators on the initial space.

Proof. For $z=\operatorname{Re} z+i \operatorname{Im} z \in \mathcal{K}_{S}$, we have

$$
W_{0}(z)=e^{i\left(a_{0}^{+}(z)+a_{0}(z)\right)}=\exp i \sqrt{2}\left(q_{0}(\operatorname{Re} z)+p_{0}(\operatorname{Im} z)\right) .
$$

Then by definition

$$
\begin{aligned}
j_{t}^{\circ}\left(W_{0}(z)\right)= & \exp i \sqrt{2}\left(\hat{j}_{t}\left(q_{0}(\operatorname{Re} z)\right)+\hat{j}_{t}\left(p_{0}(\operatorname{Im} z)\right)\right) \\
= & \exp i \sqrt{2}\left[q_{0}(\operatorname{Re} z)+p_{0}(\operatorname{Im} z)+\frac{1}{\sqrt{2}} A_{t}^{+}(T(\operatorname{Im} z)-T(i \operatorname{Re} z))\right. \\
& \left.+\frac{1}{\sqrt{2}} A_{t}(T(\operatorname{Im} z)-T(i \operatorname{Re} z))\right] \\
= & W_{0}(z) \otimes \exp i\left[-A_{t}^{+}(T(i z))-A_{t}(T(i z))\right] \\
= & W_{0}(z) \otimes W\left(-\chi_{[0, t]} \otimes T(i z)\right) .
\end{aligned}
$$

This proves the equivalence between (3.31) and (3.32). Since the Weyl operators are linearly independent, the map defined by the right-hand side of (3.32) extends by linearity to the $*$-algebra algebraically generated by them. Moreover, $j_{t}^{\circ}$ is injective because it maps Weyl operators into Weyl operators and these are linearly independent. Using (3.9) and (3.35) one has for any $z, z^{\prime} \in \mathcal{K}_{S}$ :

$$
\begin{aligned}
j_{t}^{\circ}\left(W_{0}(z) W_{0}\left(z^{\prime}\right)\right)= & e^{-i \operatorname{Im}\left\langle z, z^{\prime}\right\rangle} j_{t}^{\circ}\left(W_{0}\left(z+z^{\prime}\right)\right) \\
= & e^{-i \operatorname{Im}\left\langle z, z^{\prime}\right\rangle} W_{0}\left(z+z^{\prime}\right) \otimes \exp i\left[\left(-A_{t}^{+}(T(i z))-A_{t}(T(i z))\right)\right. \\
& \left.+\left(-A_{t}^{+}\left(T\left(i z^{\prime}\right)\right)-A_{t}\left(T\left(i z^{\prime}\right)\right)\right)\right]
\end{aligned}
$$




$$
\begin{aligned}
= & W_{0}(z) W_{0}\left(z^{\prime}\right) \otimes \exp i\left[\left(-A_{t}^{+}(T(i z))-A_{t}(T(i z))\right)\right. \\
& \left.+\left(-A_{t}^{+}\left(T\left(i z^{\prime}\right)\right)-A_{t}\left(T\left(i z^{\prime}\right)\right)\right)\right] \\
= & j_{t}^{\circ}\left(W_{0}(z)\right) j_{t}^{\circ}\left(W_{0}\left(z^{\prime}\right)\right) \exp \frac{1}{2}\left[A_{t}^{+}(T(i z))\right. \\
& \left.+A_{t}(T(i z)), A_{t}^{+}\left(T\left(i z^{\prime}\right)\right)+A_{t}\left(T\left(i z^{\prime}\right)\right)\right] \\
= & j_{t}^{\circ}\left(W_{0}(z)\right) j_{t}^{\circ}\left(W_{0}\left(z^{\prime}\right)\right) \exp \frac{1}{2}\left(\left[A_{t}^{+}(T(i z)), A_{t}\left(T\left(i z^{\prime}\right)\right)\right]\right. \\
& \left.+\left[A_{t}(T(i z)), A_{t}^{+}\left(T\left(i z^{\prime}\right)\right)\right]\right) \\
= & j_{t}^{\circ}\left(W_{0}(z)\right) j_{t}^{\circ}\left(W_{0}\left(z^{\prime}\right)\right) \exp \frac{1}{2}\left(-t\left\langle T\left(i z^{\prime}\right), T(i z)\right\rangle+t\left\langle T(i z), T\left(i z^{\prime}\right)\right\rangle\right) \\
= & j_{t}^{\circ}\left(W_{0}(z)\right) j_{t}^{\circ}\left(W_{0}\left(z^{\prime}\right)\right) \exp i t \operatorname{Im}\left\langle T(i z), T\left(i z^{\prime}\right)\right\rangle .
\end{aligned}
$$

This proves that $j_{t}^{\circ}$ is a $*$-homomorphism of associative $*$-algebras if and only if

$$
\operatorname{Im}\left\langle T(i z), T\left(i z^{\prime}\right)\right\rangle=0 ; \quad \forall z, z^{\prime} \in \mathcal{K}_{S} .
$$

Or equivalently if and only if

$$
\operatorname{Im}\left\langle T z, T z^{\prime}\right\rangle=0 ; \quad \forall z, z^{\prime} \in \mathcal{K}_{S} .
$$

In this case $j_{t}^{\circ}$ will be automatically continuous hence it extends to a $*-$ homomorphism of the whole Weyl $C^{*}$-algebra. Since the same is true for $\left(j_{t}^{\circ}\right)^{-1}$, it follows that $j_{t}^{\circ}$ is a $*$-isomorphism of the Weyl $C^{*}$-algebra $W_{0}\left(\mathcal{K}_{S}\right)$ onto its image. Finally, from Remark 3.1 we know that

$$
\hat{j_{t}}\left(E_{0}\right)=E_{0}+c t 1 ; \quad c \in \mathbb{R}
$$

and in Proposition 3.2 it is proved that $c \neq 0$ if and only if there exists $\omega \in \mathbb{C} \backslash\{0\}$ such that $\omega T$ is either a symplectic or an anti-symplectic map with respect to the symplectic form on $\mathcal{K}_{S}$ given by $\sigma\left(z, z^{\prime}\right)=\operatorname{Im}\left\langle z, z^{\prime}\right\rangle$. Then, under condition (3.34), the constant real number $c$ must be equal to zero. Therefore

$$
\hat{j}_{t}\left(E_{0}\right)=E_{0} \otimes 1=\mathbf{1}
$$

as desired.

\section{Semigroups Associated to the Heat Flows}

\subsection{Construction scheme}

Recall that the initial and noise spaces are given respectively by (3.16) and (3.17) and define

$$
j_{t}:=j_{t}^{\circ} \otimes u_{t}^{\circ}: W_{0}\left(\mathcal{K}_{S}\right) \rightarrow W_{0}\left(\mathcal{K}_{S}\right) \otimes W\left(L^{2}\left(\mathbb{R}_{+}\right) \otimes \mathcal{K}_{S}\right),
$$

where $j_{t}^{\circ}$ stands for the process defined in Theorem 3.1 and $u_{t}^{\circ}$ is the time shift defined in Sec. 2.2. 
Proposition 4.1. The family $\left\{j_{t} ; t \geq 0\right\}$ is a 1-parameter semigroup of *-homomorphisms of $\mathcal{B}\left(\mathcal{K}_{S} \otimes \Gamma\left(L^{2}\left(\mathbb{R}_{+}\right) \otimes \mathcal{K}_{S}\right)\right)$.

Proof. For $z \in \mathcal{K}_{S}, b \in \mathcal{B}\left(\Gamma\left(L^{2}\left(\mathbb{R}_{+}\right) \otimes \mathcal{K}_{S}\right)\right)$, recall that

$$
W_{0}(z)=e^{i\left(a_{0}(z)+a_{0}^{+}(z)\right)}=e^{i \sqrt{2} q_{0}(z)}
$$

and

$$
\hat{j}_{t}\left(q_{0}(z)\right)=q_{0}(z)-\frac{1}{\sqrt{2}}\left[A_{t}^{+}(T(i z))+A_{t}(T(i z))\right] .
$$

To simplify the notations let us write:

$$
\Psi_{[0, t]}(z):=-\frac{1}{\sqrt{2}}\left[A_{t}^{+}(T(i z))+A_{t}(T(i z))\right]
$$

so that

$$
\hat{j}_{t}\left(q_{0}(z)\right)=q_{0}(z)+\Psi_{[0, t]}(z) .
$$

Notice that the process $\Psi_{[0, t]}$ is additive and covariant with respect to the time shift $u_{t}^{o}$, i.e.

$$
u_{s}^{\circ}\left(\Psi_{[0, t]}(z)\right)=\Psi_{[s, s+t]}(z) .
$$

Therefore, for any $s, t \geq 0$ :

$$
\begin{aligned}
& j_{s} \circ j_{t}\left(W_{0}(z) \otimes b\right)=\left(j_{s}^{\circ} \otimes u_{s}^{\circ}\right)\left[\left(j_{t}^{\circ} \otimes u_{t}^{\circ}\right)\left(W_{0}(z) \otimes b\right)\right]=\left(j_{s}^{\circ} \otimes u_{s}^{\circ}\right)\left[\left(j_{t}^{\circ}\left(W_{0}(z)\right) \otimes u_{t}^{\circ}(b)\right)\right] \\
& =\left(j_{s}^{\circ} \otimes u_{s}^{\circ}\right)\left[\left(j_{t}^{\circ}\left(W_{0}(z)\right) \otimes 1_{[t}\right)\left(1_{t]} \otimes u_{t}^{\circ}(b)\right)\right] \\
& =\left(j_{s}^{\circ} \otimes u_{s}^{\circ}\right)\left[\left(W_{0}(z) \otimes e^{i \sqrt{2} \Psi_{[0, t]}(z)} \otimes 1_{[t}\right)\left(1_{t]} \otimes u_{t}^{\circ}(b)\right)\right] \\
& =\left(j_{s}^{\circ} \otimes u_{s}^{\circ}\right)\left[W_{0}(z) \otimes e^{i \sqrt{2} \Psi_{[0, t]}(z)} \otimes u_{t}^{\circ}(b)\right] \\
& =\left[j_{s}^{\circ}\left(W_{0}(z)\right) \otimes 1_{[s}\right]\left[1_{s]} \otimes u_{s}^{\circ}\left(1_{0} \otimes e^{i \sqrt{2} \Psi_{[0, t]}(z)} \otimes u_{t}^{\circ}(b)\right)\right] \\
& =\left(W_{0}(z) \otimes e^{i \sqrt{2} \Psi_{[0, s]}(z)} \otimes 1_{[s}\right)\left(1_{s]} \otimes e^{i \sqrt{2} \Psi_{[s, s+t]}(z)} \otimes u_{s+t}^{\circ}(b)\right) \\
& =W_{0}(z) \otimes e^{i \sqrt{2} \Psi_{[0, s+t]}(z)} \otimes u_{s+t}^{\circ}(b)=j_{s+t}^{\circ}\left(W_{0}(z)\right) \otimes u_{s+t}^{\circ}(b) \\
& =\left(j_{s+t}^{\circ} \otimes u_{s+t}^{\circ}\right)\left(W_{0}(z) \otimes b\right)=j_{s+t}\left(W_{0}(z) \otimes b\right) \text {. }
\end{aligned}
$$

By the quantum Feynman-Kac formula, ${ }^{2}$ the Markovian cocycle $\left(j_{t}\right)$ defines a Markovian semigroup on $\mathcal{B}\left(\mathcal{H}_{S}\right)$. We shall focus on the evolution $j_{t}$ and the associated semigroup

$$
P^{t}:=E_{0]} \circ j_{t}
$$

where

$$
E_{0]}: \mathcal{B}\left(\mathcal{H}_{S}\right) \otimes \mathcal{B}\left(\Gamma\left(L^{2}\left(\mathbb{R}_{+}\right) \otimes \mathcal{K}_{S}\right)\right) \rightarrow \mathcal{B}\left(\mathcal{H}_{S}\right) \equiv \mathcal{B}\left(\mathcal{H}_{S}\right) \otimes 1
$$


denote the vacuum conditional expectation (cf. Sec. 2.3), i.e.

$$
E_{0]}\left(b_{0} \otimes b\right)=\langle\Phi, b \Phi\rangle b_{0} ; \quad b_{0} \in \mathcal{B}\left(\mathcal{H}_{S}\right), \quad b \in \mathcal{B}\left(\Gamma\left(L^{2}\left(\mathbb{R}_{+}\right) \otimes \mathcal{K}_{S}\right)\right) .
$$

Proposition 4.2. In the above notations, the transformation

$$
P^{t}:=E_{0]} \circ j_{t}: W_{0}\left(\mathcal{K}_{S}\right) \rightarrow W_{0}\left(\mathcal{K}_{S}\right)
$$

is a Markovian semigroup.

Proof. Let $s, t \geq 0$ and $W_{0}(z) \in W_{0}\left(\mathcal{K}_{S}\right)$

$$
\begin{aligned}
P^{s} P^{t}\left(W_{0}(z)\right) & =E_{0]}\left[\left(j_{s}^{\circ} \otimes u_{s}^{\circ}\right)\left(E_{0]}\left(j_{t}^{\circ} \otimes u_{t}^{\circ}\right)\left(W_{0}(z)\right)\right)\right] \\
& =E_{0]}\left[\left(j_{s}^{\circ} \otimes u_{s}^{\circ}\right)\left(E_{0]}\left(j_{t}^{\circ}\left(W_{0}(z)\right) \otimes u_{t}^{\circ}(1)\right)\right)\right] \\
& =E_{0]}\left[\left(j_{s}^{\circ} \otimes u_{s}^{\circ}\right)\left(E_{0]}\left(W_{0}(z) \otimes W\left(-\chi_{[0, t]} \otimes T(i z)\right)\right)\right)\right] \\
& =E_{0]}\left(j_{s}^{\circ}\left(W_{0}(z)\right) \otimes u_{s}^{\circ}(1)\right) E_{0]}\left(W\left(-\chi_{[s, s+t]} \otimes T(i z)\right)\right) \\
& =E_{0]}\left(W_{0}(z) \otimes W\left(-\chi_{[0, s]} \otimes T(i z)\right)\right) E_{0]}\left(W\left(-\chi_{[s, s+t]} \otimes T(i z)\right)\right) \\
& =W_{0}(z) E_{0]}\left(W_{0}\left(-\chi_{[0, s]} \otimes T(i z)\right)\right) E_{0]}\left(W\left(-\chi_{[s, s+t]} \otimes T(i z)\right)\right) \\
& =W_{0}(z) E_{0]}\left(W\left(-\chi_{[0, s+t]} \otimes T(i z)\right)\right)=E_{0]}\left(j_{s+t}^{\circ} \otimes u_{s+t}^{\circ}\left(W_{0}(z)\right)\right) \\
& =P^{s+t}\left(W_{0}(z)\right),
\end{aligned}
$$

where we have used the identities (2.6) and (2.7).

Proposition 4.3. Let $P^{t}=e^{-t L}$ be the semigroup defined in Proposition 4.2. Then the Weyl operators are eigenvectors of $L$. More precisely, for any $z \in \mathcal{K}_{S}$, one has

$$
L\left(W_{0}(z)\right)=\frac{1}{2}\|T(i z)\|^{2} W_{0}(z) .
$$

Proof. For $z \in \mathcal{K}_{S}$ write

$$
W_{0}(z)=\exp i\left(a_{0}^{+}(z)+a_{0}(z)\right)=\exp i \sqrt{2} q_{0}(z),
$$

then by definition

$$
\begin{aligned}
j_{t}\left(W_{0}(z)\right) & =\exp i \sqrt{2} \hat{j}_{t}\left(q_{0}(z)\right) \\
& =W_{0}(z) \otimes \exp i\left[-A_{t}^{+}(T(i z))-A_{t}(T(i z))\right] \\
& =W_{0}(z) \otimes e^{-i A_{t}^{+}(T(i z))} e^{-i A_{t}(T(i z))} e^{\left.-\frac{1}{2} t \| T(i z)\right) \|^{2}} .
\end{aligned}
$$

Therefore

$$
P^{t}\left(W_{0}(z)\right)=\exp \left(-\frac{1}{2} t\|T(i z)\|^{2}\right) W_{0}(z) ; \quad \forall z \in \mathcal{K}_{S}
$$

and therefore, from $P^{t}=e^{-t L}$, one has

$$
L\left(W_{0}(z)\right)=\frac{1}{2}\|T(i z)\|^{2} W_{0}(z) ; \quad \forall z \in \mathcal{K}_{S} .
$$


The following proposition shows that the class of Markov generators on $W_{0}\left(\mathcal{K}_{S}\right)$ for which the Weyl operators are eigenvectors, is much larger than the class of generators of Lie algebra induced time shifts.

Proposition 4.4. Let $S(\cdot, \cdot)$ be a positive semi-definite real bilinear form on $W_{0}\left(\mathcal{K}_{S}\right)$. Then there exists a unique linear operator $L$ satisfying

$$
L\left(W_{0}(z)\right)=\frac{1}{2} S(z, z) W_{0}(z) ; \quad z \in \mathcal{K}_{S}
$$

Moreover, the 1-parameter family

$$
e^{-t L}\left(W_{0}(z)\right):=e^{-\frac{t}{2} S(z, z)} W_{0}(z) ; \quad t \geq 0, z \in \mathcal{K}_{S}
$$

is a completely positive identity preserving semigroup on $W_{0}\left(\mathcal{K}_{S}\right)$. In particular, formula (4.4) defines a Markov generator for arbitrary real linear operator $T$.

Proof. To prove complete positivity we have to show that

$$
\forall n \in \mathbb{N}, \quad \forall a_{1}, \ldots, a_{n}, b_{1}, \ldots, b_{n} \in W_{0}\left(\mathcal{K}_{S}\right)
$$

one has

$$
\sum_{i, j=1}^{n} b_{i}^{*} e^{-t L}\left(a_{i}^{*} a_{j}\right) b_{j} \geq 0 .
$$

Let $n \in \mathbb{N}$. For $1 \leq i \leq n$ take

$$
b_{i}=\lambda_{i} W_{0}\left(u_{i}\right) ; \quad a_{i}=\mu_{i} W_{0}\left(z_{i}\right),
$$

where $\lambda_{i}, \mu_{i} \in \mathbb{C}$ and $u_{i}, z_{i} \in \mathcal{K}_{S}$. In these notations one has

$$
\begin{aligned}
b_{i}^{*} e^{-t L} & \left(a_{i}^{*} a_{j}\right) b_{j} \\
& =\bar{\lambda}_{i} W_{0}\left(u_{i}\right)^{*} e^{-t L}\left(\bar{\mu}_{i} W_{0}\left(z_{i}\right)^{*} W_{0}\left(z_{j}\right) \mu_{j}\right) \lambda_{j} W_{0}\left(u_{j}\right) \\
& =\bar{\lambda}_{i} W_{0}\left(u_{i}\right)^{*} \bar{\mu}_{i} e^{i \sigma\left(z_{i}, z_{j}\right)} \exp \left(-\frac{t}{2} S\left(z_{j}-z_{i}, z_{j}-z_{i}\right)\right) W_{0}\left(-z_{i}+z_{j}\right) \mu_{j} \lambda_{j} W_{0}\left(u_{j}\right) \\
& =\exp \left(-\frac{t}{2} S\left(z_{j}-z_{i}, z_{j}-z_{i}\right)\right) \bar{\lambda}_{i} W_{0}\left(u_{i}\right)^{*} \bar{\mu}_{i} W_{0}\left(z_{i}\right)^{*} W_{0}\left(z_{j}\right) \mu_{j} \lambda_{j} W_{0}\left(u_{j}\right) \\
& =\exp \left(-\frac{t}{2} S\left(z_{j}-z_{i}, z_{j}-z_{i}\right)\right)\left(a_{i} b_{i}\right)^{*}\left(a_{j} b_{j}\right) .
\end{aligned}
$$

Thus it is sufficient to prove that

$$
\exp \left(-\frac{t}{2} S\left(z_{j}-z_{i}, z_{j}-z_{i}\right)\right)
$$

is positive definite and this follows from Schur's lemma. 


\subsection{The associated quantum diffusion}

Let $\mathcal{K}_{S}$ be a complex separable Hilbert space and $g=\left(g_{j}\right)_{j=1}^{\infty}$ be an orthonormal basis of $\mathcal{K}_{S, r}$. For $z=\sum_{j} \lambda_{j} g_{j} \in \mathcal{K}_{S}, \lambda_{j} \in \mathbb{C}$, define

$$
\begin{gathered}
\operatorname{Re} \lambda_{n}:=\operatorname{Re}\left\langle g_{n}, z\right\rangle=\left\langle g_{n}, \operatorname{Re} z\right\rangle, \\
\operatorname{Im} \lambda_{n}:=\operatorname{Im}\left\langle g_{n}, z\right\rangle=\left\langle g_{n}, \operatorname{Im} z\right\rangle, \\
\operatorname{Re} z:=\sum_{k} \operatorname{Re}\left(\lambda_{k}\right) g_{k} \in \mathcal{K}_{S, r} ; \quad \operatorname{Im} z:=\sum_{k} \operatorname{Im}\left(\lambda_{k}\right) g_{k} \in \mathcal{K}_{S, r} .
\end{gathered}
$$

Then one has:

$$
\begin{aligned}
& a_{0}^{+}(z)=a_{0}^{+}\left(\sum_{j} \lambda_{j} g_{j}\right):=\sum_{j} \lambda_{j} a_{0}^{+}\left(g_{j}\right), \\
& a_{0}(z)=a_{0}\left(\sum_{j} \lambda_{j} g_{j}\right):=\sum_{j} \bar{\lambda}_{j} a_{0}\left(g_{j}\right) \\
& p_{0}(z)=\sum_{j=1}^{\infty} \frac{1}{i \sqrt{2}}\left(\bar{\lambda}_{j} a_{0}\left(g_{j}\right)-\lambda_{j} a_{0}^{+}\left(g_{j}\right)\right), \\
& q_{0}(z)=\sum_{j=1}^{\infty} \frac{1}{\sqrt{2}}\left(\bar{\lambda}_{j} a_{0}\left(g_{j}\right)+\lambda_{j} a_{0}^{+}\left(g_{j}\right)\right),
\end{aligned}
$$

where all series are strongly convergent on the maximal algebraic domain. Therefore

$$
\begin{aligned}
W_{0}(z) & :=\exp i\left(a_{0}^{+}(z)+a_{0}(z)\right)=\exp i\left(\sum_{j} \lambda_{j} a_{0}^{+}\left(g_{j}\right)+\overline{\lambda_{j}} a_{0}\left(g_{j}\right)\right) \\
& =\exp i \sqrt{2}\left[\left(\sum_{j} \operatorname{Re}\left(\lambda_{j}\right) q_{0}\left(g_{j}\right)\right)+\left(\sum_{j} \operatorname{Im}\left(\lambda_{j}\right) p_{0}\left(g_{j}\right)\right)\right] \\
& =\exp i \sqrt{2}\left[q_{0}(\operatorname{Re} z)+p_{0}(\operatorname{Im} z)\right] .
\end{aligned}
$$

The following commutation relations are well known and we include the proof for completeness.

Lemma 4.1. For $z, z^{\prime} \in \mathcal{K}_{S}$ we have

$$
\begin{aligned}
{\left[a_{0}\left(z^{\prime}\right), W_{0}(z)\right] } & =i\left\langle z^{\prime}, z\right\rangle W_{0}(z), \\
{\left[a_{0}^{+}\left(z^{\prime}\right), W_{0}(z)\right] } & =-i\left\langle z, z^{\prime}\right\rangle W_{0}(z), \\
{\left[p_{0}\left(z^{\prime}\right), W_{0}(z)\right] } & =\sqrt{2} \operatorname{Re}\left\langle z^{\prime}, z\right\rangle W_{0}(z), \\
{\left[q_{0}\left(z^{\prime}\right), W_{0}(z)\right] } & =-\sqrt{2} \operatorname{Im}\left\langle z^{\prime}, z\right\rangle W_{0}(z) .
\end{aligned}
$$


Proof. By definition for any $z \in \mathcal{K}_{S}$ one has:

$$
W_{0}(z)=e^{i\left(a_{0}^{+}(z)+a_{0}(z)\right)}=e^{i a_{0}^{+}(z)} e^{i a_{0}(z)} e^{-\frac{1}{2}\|z\|^{2}},
$$

moreover

$$
\left[a_{0}\left(z^{\prime}\right), e^{i a_{0}^{+}(z)}\right]=i\left\langle z^{\prime}, z\right\rangle e^{i a_{0}^{+}(z)} ; \quad\left[a_{0}^{+}\left(z^{\prime}\right), e^{i a_{0}(z)}\right]=-i\left\langle z, z^{\prime}\right\rangle e^{i a_{0}(z)} .
$$

Hence, using Leibnitz formula, one get

$$
\begin{aligned}
{\left[a_{0}\left(z^{\prime}\right), W_{0}(z)\right] } & =\left[a_{0}\left(z^{\prime}\right), e^{i a_{0}^{+}(z)} e^{i a_{0}(z)}\right] e^{-\frac{1}{2}\|z\|^{2}} \\
& =\left(\left[a_{0}\left(z^{\prime}\right), e^{i a_{0}^{+}(z)}\right] e^{i a_{0}(z)}+e^{i a_{0}^{+}(z)}\left[a_{0}\left(z^{\prime}\right), e^{i a_{0}(z)}\right]\right) e^{-\frac{1}{2}\|z\|^{2}} \\
& =i\left\langle z^{\prime}, z\right\rangle W_{0}(z) .
\end{aligned}
$$

In a similar way, we obtain the second identity. For $z, z^{\prime} \in \mathcal{K}_{S}$ we have

$$
\begin{aligned}
{\left[p_{0}\left(z^{\prime}\right), W_{0}(z)\right] } & =\left[\frac{1}{i \sqrt{2}}\left(a_{0}\left(z^{\prime}\right)-a_{0}^{+}\left(z^{\prime}\right)\right), W_{0}(z)\right] \\
& =\frac{1}{i \sqrt{2}}\left(\left[a_{0}\left(z^{\prime}\right), W_{0}(z)\right]-\left[a_{0}^{+}\left(z^{\prime}\right), W_{0}(z)\right]\right) \\
& =\frac{1}{i \sqrt{2}}\left(i\left\langle z^{\prime}, z\right\rangle+i\left\langle z, z^{\prime}\right\rangle\right) W_{0}(z) \\
& =\sqrt{2} \operatorname{Re}\left\langle z^{\prime}, z\right\rangle W_{0}(z) .
\end{aligned}
$$

Similarly, one has

$$
\begin{aligned}
{\left[q_{0}\left(z^{\prime}\right), W_{0}(z)\right] } & =\left[\frac{1}{\sqrt{2}}\left(a_{0}\left(z^{\prime}\right)+a_{0}^{+}\left(z^{\prime}\right)\right), W_{0}(z)\right] \\
& =\frac{1}{\sqrt{2}}\left(\left[a_{0}\left(z^{\prime}\right), W_{0}(z)\right]+\left[a_{0}^{+}\left(z^{\prime}\right), W_{0}(z)\right]\right) \\
& =\frac{1}{\sqrt{2}}\left(i\left\langle z^{\prime}, z\right\rangle-i\left\langle z, z^{\prime}\right\rangle\right) W_{0}(z) \\
& =-\sqrt{2} \operatorname{Im}\left\langle z^{\prime}, z\right\rangle W_{0}(z) .
\end{aligned}
$$

Theorem 4.1. The algebraic stochastic process $j=\left\{j_{t} ; t \geq 0\right\}$ acting on $W_{0}\left(\mathcal{K}_{S}\right)$, given by (4.1), satisfies the following classical diffusion equation

$$
\begin{aligned}
d j_{t}(a)= & \sum_{k} j_{t}\left(\frac{1}{i \sqrt{2}}\left[p_{0}\left(g_{k}\right), a\right]\right) d W_{t}\left(T\left(i g_{k}\right)\right) \\
& +\sum_{k} j_{t}\left(\frac{1}{i \sqrt{2}}\left[q_{0}\left(g_{k}\right), a\right]\right) d W_{t}\left(T\left(g_{k}\right)\right)-j_{t}(L(a)) d t
\end{aligned}
$$

where $g=\left(g_{k}\right)$ is an arbitrary orthonormal basis of $\mathcal{K}_{S, r}, L$ is the generator of the semigroup $P^{t}$ defined in Proposition 4.2 and

$$
d W_{t}=d A_{t}^{+}+d A_{t} .
$$


Proof. From (4.5) we have, for any $z \in \mathcal{K}_{S}$ :

$$
j_{t}\left(W_{0}(i z)\right)=W_{0}(i z) \otimes e^{i A_{t}^{+}(T z)} e^{i A_{t}(T z)} e^{\left.-\frac{1}{2} t \| T z\right) \|^{2}}
$$

To deduce the equation for $j_{t}\left(W_{0}(i z)\right)$ it is convenient to introduce white noise notations:

$$
A_{t}^{ \pm}(F)=\int_{0}^{t} a_{s}^{ \pm}(F) d s .
$$

Since the right-hand side of (4.16) is normally ordered, its equation is obtained taking $t$-derivative and using the usual Leibnitz rule. This gives the normally ordered white noise equation:

$$
\partial_{t} j_{t}\left(W_{0}(i z)\right)=i a_{t}^{+}(T z) j_{t}\left(W_{0}(i z)\right)+i j_{t}\left(W_{0}(i z)\right) a_{t}(T z)-\frac{1}{2}\|T z\|^{2} j_{t}\left(W_{0}(i z)\right)
$$

which is equivalent to the quantum stochastic differential equation

$$
\begin{aligned}
d j_{t}\left(W_{0}(i z)\right) & =\left(i d A_{t}^{+}(T z)+i d A_{t}(T z)-\frac{1}{2}\|T z\|^{2}\right) j_{t}\left(W_{0}(i z)\right) \\
& =\left(i d W_{t}(T z)-\frac{1}{2}\|T z\|^{2}\right) j_{t}\left(W_{0}(i z)\right),
\end{aligned}
$$

where we have introduced the classical Wiener process $d W_{t}:=d A_{t}^{+}+d A_{t}$. This can be written as a classical Evans-Hudson flow introducing coordinates. Namely, writing, for $z=\sum_{k} \lambda_{k} g_{k} \in \mathcal{K}_{S}$,

$$
\begin{aligned}
z & =\sum_{k} \operatorname{Re}\left(\lambda_{k}\right) g_{k}+\sum_{k} \operatorname{Im}\left(\lambda_{k}\right)\left(i g_{k}\right) \\
& =\sum_{k}\left\langle g_{k}, \operatorname{Re}\left(\lambda_{k}\right)\right\rangle g_{k}+\sum_{k}\left\langle g_{k}, \operatorname{Im}\left(\lambda_{k}\right)\right\rangle\left(i g_{k}\right)
\end{aligned}
$$

then

$$
T z=\sum_{k}\left\langle g_{k}, \operatorname{Re}\left(\lambda_{k}\right)\right\rangle T\left(g_{k}\right)+\sum_{k}\left\langle g_{k}, \operatorname{Im}\left(\lambda_{k}\right)\right\rangle T\left(i g_{k}\right)
$$

and the stochastic differential equation becomes:

$$
\begin{aligned}
d j_{t}\left(W_{0}(z)\right)= & i \sum_{k}\left\langle g_{k}, \operatorname{Im} z\right\rangle j_{t}\left(W_{0}(z)\right) d W_{t}\left(T\left(g_{k}\right)\right) \\
& -i \sum_{k}\left\langle g_{k}, \operatorname{Re} z\right\rangle j_{t}\left(W_{0}(z)\right) d W_{t}\left(T\left(i g_{k}\right)\right)-\frac{1}{2}\|T(i z)\|^{2} j_{t}\left(W_{0}(z)\right) d t .
\end{aligned}
$$

Then, using (4.13), (4.14), we obtain

$$
\begin{aligned}
d j_{t}\left(W_{0}(z)\right)= & \sum_{k} j_{t}\left(\frac{1}{i \sqrt{2}}\left[q_{0}\left(g_{k}\right), W_{0}(z)\right]\right) d W_{t}\left(T\left(g_{k}\right)\right) \\
& +\sum_{k} j_{t}\left(\frac{1}{i \sqrt{2}}\left[p_{0}\left(g_{k}\right), W_{0}(z)\right]\right) d W_{t}\left(T\left(i g_{k}\right)\right)-j_{t}\left(L\left(W_{0}(z)\right)\right) d t
\end{aligned}
$$

and this proves the statement. 


\section{Quantum Laplacians}

The classical $d$-dimensional Laplacian can be characterized as the only second order differential operator on $\mathbb{R}^{d}$ which annihilates the multiples of the identity and is invariant under the Euclidean (translations and rotations) group. In order to extend this notion to a general $*$-algebra $\mathcal{A}$ one has to extend to this context:

(i) the notion of second order differential operator;

(ii) the notion of Euclidean group.

It is natural to add the further requirements:

(iii) there should exist (at least) one abelian $*$-sub-algebra of $\mathcal{A}$, isomorphic to $C^{\infty}\left(\mathbb{R}^{d}\right)$, such that the restriction of the quantum Laplacian to this sub-algebra coincides with the usual Laplacian on $\mathbb{R}^{d}$;

(iv) the quantum Laplacian should be the generator of a quantum Markov semigroup.

In the classical case these requirements are not independent. In the quantum case they are. Moreover, in the quantum case these requirements can be realized in a multiplicity of different ways (cf. for example Refs. 5 and $9, \ldots$ ) and the best way to discriminate among the various possibilities is to accumulate a sufficiently large class of interesting examples against which to check the general definitions.

In this paper we investigate this problem in the special context in which the *-algebra $\mathcal{A}$ is the Fock representation of the Weyl algebra over a 1-particle space of the form (3.7). In this context the Weyl operators are the quantum analogue of the trigonometric exponentials hence the Markov generators $L=L_{T}$ characterized by (4.4) are natural candidates for the role of second order differential operators. Among them we want to single out the natural Laplacians in terms of an invariance property with respect to the action of a group which generalizes the usual Euclidean group.

The natural symmetry group of the Weyl algebra is the symplectic group associated to a symplectic form $\sigma$.

Definition 5.1. Let $F$ be a real Hilbert space and let $\sigma(\cdot, \cdot)$ be a nondegenerate symplectic form on $F$. The group of real linear maps $R$ on $F$ leaving $\sigma$ invariant, i.e.

$$
\sigma\left(R z, R z^{\prime}\right)=\sigma\left(z, z^{\prime}\right) ; \quad \forall z, z^{\prime} \in F
$$

is called the $\sigma$-symplectic group on $F$ and is denoted $O_{\sigma}(F)$.

In this paper (according to the notations of the previous sections) we start from a complex Hilbert space $\mathcal{K}_{S}$ and consider it as a real Hilbert space with real scalar product given by the real part of the original scalar product $\langle\cdot, \cdot\rangle \equiv\langle\cdot, \cdot\rangle_{\mathcal{K}_{S}}$ :

$$
\langle f, g\rangle_{r}:=\operatorname{Re}\langle f, g\rangle
$$


and with a nondegenerate symplectic form given by the imaginary part of the original scalar product:

$$
\sigma\left(z, z^{\prime}\right):=\operatorname{Im}\left\langle z, z^{\prime}\right\rangle .
$$

The corresponding symplectic group on $\mathcal{K}_{S}$ will be denoted $O_{r, \sigma}\left(\mathcal{K}_{S}\right)$.

To any $R \in O_{r, \sigma}\left(\mathcal{K}_{S}\right)$ we associate a linear map $\alpha_{R}$ on $W_{0}\left(\mathcal{K}_{S}\right)$ characterized by:

$$
\alpha_{R}\left(W_{0}(z)\right)=W_{0}(R z) ; \quad \forall z \in \mathcal{K}_{S} .
$$

Such a map is well defined by the linear independence of the Weyl operators and one easily checks that:

(i) it is an automorphism in $\operatorname{Aut}\left(W_{0}\left(\mathcal{K}_{S}\right)\right)$;

(ii) the map $R \mapsto \alpha_{R}$ is an injective group homomorphism from $O_{r, \sigma}\left(\mathcal{K}_{S}\right)$ to $\operatorname{Aut}\left(W_{0}\left(\mathcal{K}_{S}\right)\right)$.

Remark 5.1. The following lemma shows that $O_{r, \sigma}\left(\mathcal{K}_{S}\right)$ contains the usual Hilbert space "rotations", i.e. the group $U n\left(\mathcal{K}_{S}\right)$ of (complex) unitary operators on $\mathcal{K}_{S}$.

Proposition 5.1. In the above notations, the following inclusion holds

$$
U n\left(\mathcal{K}_{S}\right) \subseteq O_{r, \sigma}\left(\mathcal{K}_{S}\right)
$$

Conversely, an element $R \in O_{r, \sigma}\left(\mathcal{K}_{S}\right)$ lies in $U n\left(\mathcal{K}_{S}\right)$ if and only if

$$
i z=R^{-1} i R z ; \quad \forall z \in \mathcal{K}_{S} .
$$

Proof. If $R \in U n\left(\mathcal{K}_{S}\right)$ then, for any $z, z^{\prime} \in \mathcal{K}_{S}$, we have

$$
\sigma\left(R z, R z^{\prime}\right)=\operatorname{Im}\left\langle R z, R z^{\prime}\right\rangle=\operatorname{Im}\left\langle z, z^{\prime}\right\rangle=\sigma\left(z, z^{\prime}\right) .
$$

Hence $R \in O_{r, \sigma}\left(\mathcal{K}_{S}\right)$. Conversely, assume that $R \in O_{r, \sigma}\left(\mathcal{K}_{S}\right)$. Then $R \in \operatorname{Un}\left(\mathcal{K}_{S}\right)$ if and only if

$$
\left\langle R z, R z^{\prime}\right\rangle=\left\langle z, z^{\prime}\right\rangle ; \quad \forall z, z^{\prime} \in \mathcal{K}_{S} .
$$

On the other hand, from the algebraic identity (cf. (5.2))

$$
\left\langle z, z^{\prime}\right\rangle=\sigma\left(z, i z^{\prime}\right)+i \sigma\left(z, z^{\prime}\right) ; \quad z, z^{\prime} \in \mathcal{K}_{S},
$$

we deduce that

$$
\left\langle R z, R z^{\prime}\right\rangle=\sigma\left(R z, i R z^{\prime}\right)+i \sigma\left(R z, R z^{\prime}\right)=\sigma\left(R z, i R z^{\prime}\right)+i \sigma\left(z, z^{\prime}\right) .
$$

Thus, using (5.1), we see that $R \in U n\left(\mathcal{K}_{S}\right)$ if and only if

$$
\sigma\left(z, i z^{\prime}\right)=\sigma\left(R z, i R z^{\prime}\right)=\sigma\left(z, R^{-1} i R z^{\prime}\right) ; \quad \forall z, z^{\prime} \in \mathcal{K}_{S} .
$$

Since $\sigma$ is nondegenerate this is equivalent to

$$
i z^{\prime}=R^{-1} i R z^{\prime} ; \quad \forall z^{\prime} \in \mathcal{K}_{S}
$$

and this completes the proof. 
Definition 5.2. A linear operator $L: W_{0}\left(\mathcal{K}_{S}\right) \rightarrow W\left(\mathcal{K}_{S}\right)$ is said to be $O_{r, \sigma}\left(\mathcal{K}_{S}\right)$ invariant if

$$
L \circ \alpha_{R}=\alpha_{R} \circ L ; \quad \forall R \in O_{r, \sigma}\left(\mathcal{K}_{S}\right) .
$$

Proposition 5.2. Let $L=L_{T}$ be any Markov generator satisfying (4.4). Then $L_{T}$ is $O_{r, \sigma}\left(\mathcal{K}_{S}\right)$-invariant if and only if $T$ satisfies

$$
\|T z\|=\|T R(z)\| ; \quad \forall R \in O_{r, \sigma}\left(\mathcal{K}_{S}\right), \quad \forall z \in \mathcal{K}_{S} .
$$

Moreover, (5.5) holds if and only if, denoting $T^{*}$ the adjoint of $T$ in the real Hilbert space $\left\{\mathcal{K}_{S},\langle\cdot, \cdot\rangle_{r}\right\}, T^{*} T$ is a positive multiple of the identity operator on $\mathcal{K}_{S}$.

Proof. From (4.4) it follows that, for any $z \in \mathcal{K}_{S}$,

$$
L\left(\alpha_{R}\left(W_{0}(z)\right)\right)=L\left(W_{0}(R z)\right)=\frac{1}{2}\|T(i R z)\|^{2} W_{0}(R z) ; \quad \forall R \in O_{r, \sigma}\left(\mathcal{K}_{S}\right) .
$$

On the other hand, for any $R \in O_{r, \sigma}\left(\mathcal{K}_{S}\right)$,

$$
\alpha_{R}\left(L\left(W_{0}(z)\right)\right)=\alpha_{R}\left(\frac{1}{2}\|T(i z)\|^{2} W_{0}(z)\right)=\frac{1}{2}\|T(i z)\|^{2} W_{0}(R z) .
$$

The rotation invariance condition (5.4) is then equivalent to

$$
\|T(i R z)\|^{2}=\|T(i z)\|^{2} ; \quad \forall R \in O_{r, \sigma}\left(\mathcal{K}_{S}\right) .
$$

Since the original scalar product $\langle\cdot, \cdot\rangle$ on $\mathcal{K}_{S}$ gives the same norm as $\langle\cdot, \cdot\rangle_{r}$, this is equivalent to

$$
\left\langle R^{*}\left(T^{*} T\right) R z, z\right\rangle_{r}=\left\langle T^{*} T z, z\right\rangle_{r} ; \quad \forall z \in \mathcal{K}_{S}, \quad \forall R \in O_{r, \sigma}\left(\mathcal{K}_{S}\right),
$$

or equivalently

$$
R^{*}\left(T^{*} T\right) R=T^{*} T ; \quad \forall R \in O_{r, \sigma}\left(\mathcal{K}_{S}\right) .
$$

Finally, a standard argument shows that this is equivalent to

$$
T^{*} T=\lambda I
$$

for some positive real number $\lambda$, where $I$ stands for the identity operator on $\mathcal{K}_{S}$

Remark. Proposition 5.2 and identity (4.4) imply that the action of an $O_{r, \sigma}\left(\mathcal{K}_{S}\right)$ invariant generator $L_{T}$ on the Weyl operators depend on $T$ only through a multiplicative constant. Choosing this constant equal to 1 (i.e. $T$ isometric) we fix a unique generator independent of $T$. This justifies the following definition.

Definition 5.3. The quantum Laplacian on $W_{0}\left(\mathcal{K}_{S}\right)$ is the unique Markov generator $\Delta^{Q}$ on $W_{0}\left(\mathcal{K}_{S}\right)$ characterized by:

$$
\Delta^{Q}\left(W_{0}(z)\right)=\frac{1}{2}\|z\|^{2} W_{0}(z) ; \quad z \in \mathcal{K}_{S}
$$


Proposition 5.3. The generator $L_{T}$ of a nontrivial (i.e. not the identity) Lie algebra time shift cannot be $O_{r, \sigma}\left(\mathcal{K}_{S}\right)$-invariant.

Proof. By Proposition 5.2 the $O_{r, \sigma}\left(\mathcal{K}_{S}\right)$-invariance of a generator $L_{T}$ is characterized by the existence of a real number $\lambda$ such that $T^{*} T=\lambda I$. If the time shift, associated to $L_{T}$, is not the identity, then $\lambda>0$. In particular $T$ is bounded invertible, hence its range is the whole of $\mathcal{K}_{S}$. But, due to (5.2), this is incompatible with the condition

$$
\operatorname{Im}\left\langle T z, T z^{\prime}\right\rangle=0 ; \quad \forall z, z^{\prime} \in \mathcal{K}_{S}
$$

which, according to Theorem 3.1, is necessary for $L_{T}$ to be associated to a Lie algebra time shift.

Remark 5.2. The above result shows that, if we want the quantum Brownian motion to be canonically associated to a notion of "quantum Laplacian", then we have to relax at least one of the following two requirements:

(i) the Markov generator associated to the time shift is $O_{r, \sigma}\left(\mathcal{K}_{S}\right)$-invariant;

(ii) the time shift is of Lie algebra type.

Definition 5.3 pursues the latter alternative. The proposition below shows that, in this case, the quantum Laplacian has a very strong extension property.

Proposition 5.4. Let $\mathcal{K}_{S}=\mathcal{K}_{S, r} \dot{+} \mathcal{K}_{S, r}$ be a complex Hilbert space with real scalar product, $L=L_{T}$ be a linear operator on $W_{0}\left(\mathcal{K}_{S}\right)$ satisfying (4.4) for some real linear operator $T$ on $\mathcal{K}_{S}$ and $g=\left(g_{k}\right)_{k}$ be any orthonormal basis of $\mathcal{K}_{S, r}$. Then the generator $L$ of the semigroup $P^{t}$ introduced in (4.2) is a second order, constant coefficients, differential operator characterized by

$$
\begin{aligned}
L\left(W_{0}(z)\right)= & \frac{1}{4} \sum_{k}\left[q_{0}\left(T^{*}\left(g_{k}\right)\right),\left[q_{0}\left(T^{*}\left(g_{k}\right)\right), W_{0}(z)\right]\right] \\
& +\frac{1}{4} \sum_{k}\left[p_{0}\left(T^{*}\left(g_{k}\right)\right),\left[p_{0}\left(T^{*}\left(g_{k}\right)\right), W_{0}(z)\right]\right] ; \quad \forall z \in \mathcal{K}_{S} .
\end{aligned}
$$

Furthermore, if $L$ is the quantum Laplacian $\Delta^{Q}$, one can choose $T=I$ in (5.7) whenever $T$ maps $\mathcal{K}_{S, r}$ into itself. In this case the restrictions of $L$ to the (Abelian) polynomial algebras generated respectively by the position and momentum fields, coincide with the infinitesimal generator of the classical Wiener process, i.e. with the usual Volterra Laplacian $\Delta_{V}$.

Remark. Proposition 5.4 shows that the restrictions of $\Delta^{Q}$ to both Abelian algebras $L^{\infty}\left(q_{0}\right)$ (the "position algebra") and $L^{\infty}\left(p_{0}\right)$ (the "momentum algebra") coincide with the classical Volterra Laplacian $\Delta_{V}$. Moreover, Theorem 4.1, combined with the Hudson-Parthasarathy theory shows that the flow, giving rise to 
$\Delta^{Q}$, can be unitarily implemented. However the Remark 5.2 shows that it cannot be a Lie algebra type time shift.

Proof. Denoting, for $z, z^{\prime} \in \mathcal{K}_{S}$,

$$
\left\langle z, z^{\prime}\right\rangle_{r}:=\operatorname{Re}\left\langle z, z^{\prime}\right\rangle=\left\langle\operatorname{Re} z, \operatorname{Re} z^{\prime}\right\rangle+\left\langle\operatorname{Im} z, \operatorname{Im} z^{\prime}\right\rangle ; \quad \forall z, z^{\prime} \in \mathcal{K}_{S},
$$

one has

$$
\|z\|_{r}^{2}:=\langle z, z\rangle_{r}=\|\operatorname{Re} z\|^{2}+\|\operatorname{Im} z\|^{2}=\|z\|^{2} .
$$

We know that the generator of the semigroup $P^{t}$ defined in (4.3) is given by

$$
\begin{aligned}
L\left(W_{0}(z)\right) & =\frac{1}{2}\|T(i z)\|^{2} W_{0}(z)=\frac{1}{2}\left(\|\operatorname{Re} T(i z)\|^{2}+\|\operatorname{Im} T(i z)\|^{2}\right) W_{0}(z) \\
& =\frac{1}{2} \sum_{k}\left(\left\langle g_{k}, \operatorname{Re} T(i z)\right\rangle_{r}^{2}+\left\langle g_{k}, \operatorname{Im} T(i z)\right\rangle_{r}^{2}\right) W_{0}(z) \\
& =\frac{1}{2} \sum_{k}\left[\left(\operatorname{Re}\left\langle g_{k}, T(i z)\right\rangle\right)^{2}+\left(\operatorname{Im}\left\langle g_{k}, T(i z)\right\rangle\right)^{2}\right] W_{0}(z) \\
& =\frac{1}{2} \sum_{k}\left[\left(\operatorname{Re}\left\langle T^{*}\left(g_{k}\right), z\right\rangle\right)^{2}+\left(\operatorname{Im}\left\langle T^{*}\left(g_{k}\right), z\right\rangle\right)^{2}\right] W_{0}(z)
\end{aligned}
$$

where $T^{*}$ is the $\langle\cdot, \cdot\rangle_{r}$-adjoint of $T$. Then, by using Lemma 4.1, we have

$$
\begin{aligned}
L\left(W_{0}(z)\right)= & \frac{1}{4} \sum_{k}\left[q_{0}\left(T^{*}\left(g_{k}\right)\right),\left[q_{0}\left(T^{*}\left(g_{k}\right)\right), W_{0}(z)\right]\right] \\
& +\frac{1}{4} \sum_{k}\left[p_{0}\left(T^{*}\left(g_{k}\right)\right),\left[p_{0}\left(T^{*}\left(g_{k}\right)\right), W_{0}(z)\right]\right] .
\end{aligned}
$$

This proves (5.7). If $L$ is the quantum Laplacian and if $T$ maps $\mathcal{K}_{S, r}$ into itself, then $T g:=\left(T g_{k}\right)_{k}$ is also an orthonormal basis of $\mathcal{K}_{S, r}$ and one can choose $T=I$ in (5.7). The last statement follows from the identity:

$$
\left[p_{0}\left(g_{k}\right), \varphi\left(q_{0}\right)(\xi)\right]=\frac{1}{i} \partial_{\xi_{k}} \varphi\left(q_{0}\right)(\xi) ; \quad \xi_{k}:=\sqrt{2}\left\langle g_{k}, \xi\right\rangle
$$

which follows from Lemma 4.1 and is valid for any $k$ and for any smooth cylindrical function (in the $\left(g_{k}\right)$-basis) of the form $\varphi\left(q_{0}(\xi)\right), \xi \in \mathcal{K}_{S, r}$. Using multiple polarization, any element of the polynomial algebra generated by the $q_{0}(\xi)$ with $\xi \in \mathcal{K}_{S, r}$ can be expressed as a linear combination of polynomial of the form $\varphi\left(q_{0}(\xi)\right)$. This implies that

$$
\left[p_{0}\left(g_{k}\right),\left[p_{0}\left(g_{k}\right), \varphi\left(q_{0}(\xi)\right)\right]\right]=\partial_{i \xi_{k}}^{2} \varphi\left(q_{0}(\xi)\right)
$$

and therefore,

$$
\Delta^{Q}\left(\varphi\left(q_{0}(\xi)\right)\right)=\frac{1}{4} \sum_{k}\left(\partial_{i q_{k}}^{2} \varphi\right)\left(q_{0}(\xi)\right)=\frac{1}{4}\left(\Delta_{V} \varphi\right)\left(q_{0}(\xi)\right)
$$


which shows that the restriction of the quantum Laplacian to the position algebra coincides with the Volterra Laplacian on the smooth cylindrical functions. Exchanging the roles of $p_{0}$ and $q_{0}$, the above considerations are still valid. This completes the proof.

\section{Quantum Lévy-Laplacian}

In this section we construct a quantum extension of the Lévy-Laplacian in the sense of Definition 5.3. We prove that the construction, used for the Volterra Laplacian, can be repeated with the only difference that now the space $\mathcal{K}_{S}$ has to be a suitable extension of the Cesàro Hilbert space. This notion will be briefly recalled in Sec. 6.1. For more details we refer to our previous paper ${ }^{3}$ and the references cited therein.

\subsection{The Cesàro Hilbert space}

Denote $E=S(\mathbb{R})$ the space of rapidly decreasing functions on $\mathbb{R}$ so that $E^{*}=$ $S^{\prime}(\mathbb{R})$ is the space of tempered distributions on $\mathbb{R}$. The standard number operator $D=-(d / d u)^{2}+u^{2}+1$ is a densely defined self-adjoint operator on $L^{2}(\mathbb{R})$ and there exists an orthonormal basis $e=\left(e_{n}\right)_{n}$ for $L^{2}(\mathbb{R})$ such that $D e_{n}=2(n+1) e_{n}$. We define the norm $|\cdot|_{p}$ by $|f|_{p}=\left|D^{p} f\right|_{0}$ for $f \in E$ and $p \in \mathbb{R}$, where $|\cdot|_{0}$ is the $L^{2}(\mathbb{R})$-norm, and let $E_{p}$ be the completion of $E$ with respect to the norm $|\cdot|_{p}$. Then $E_{p}$ is a real separable Hilbert space with the norm $|\cdot|_{p}$ and the dual space $E_{p}^{\prime}$ of $E_{p}$ is the same as $E_{-p}$ (see Ref. 16). The space $E$ is the projective limit of $\left\{E_{p} ; p \geq 0\right\}$ and $E^{*}$ is the inductive limit of $\left\{E_{-p} ; p \geq 0\right\}$. Then $E$ becomes a nuclear space with the real standard triple

$$
E \subset H \subset E^{*}
$$

where $H \equiv L^{2}(\mathbb{R})$. We denote the complexifications of $H, E$ and $E_{p}$ by $K, N$ and $N_{p}$, respectively. i.e.

$$
N:=E \dot{+} i E ; \quad K=H+i H ; \quad N_{p}:=N_{p} \dot{+} i N_{p} ; \quad N^{*}=E^{*} \dot{+} i E^{*} .
$$

Then we obtain the complex standard triple

$$
N \subset K \subset N^{*}
$$

The scalar product $\langle\cdot, \cdot\rangle$ of $K$ will be assumed to be linear on the right.

Definition 6.1. For $f, g \in N^{*}$, the Cesàro scalar product $\langle g, f\rangle_{c}$ is defined by

$$
\langle g, f\rangle_{c}:=\lim _{n \rightarrow \infty} \frac{1}{n} \operatorname{Tr}\left(P_{n]}|f\rangle\langle g| P_{n]}\right)=\lim _{n \rightarrow+\infty} \frac{1}{n} \sum_{j=1}^{n}\left\langle g, e_{j}\right\rangle\left\langle e_{j}, f\right\rangle
$$

whenever the limit exists (on a proper space), where $\langle\cdot, \cdot\rangle$ is the canonical $\mathbb{C}$-bilinear form on $N^{*} \times N,\left\langle e_{j}, f\right\rangle=\overline{\left\langle f, e_{j}\right\rangle}$, 


$$
\begin{gathered}
|f\rangle\langle g|: x \in N \rightarrow\langle g, x\rangle f \in N^{*}, \\
P_{n]}:=\sum_{j=1}^{n}\left|e_{j}\right\rangle\left\langle e_{j}\right|
\end{gathered}
$$

and $\operatorname{Tr}$ is the usual trace on operators.

Let $S$ be the interval $(0, \pi)$. For any $\lambda \in S$, let $H_{\lambda}$ denotes the complex vector subspace of $K$ algebraically generated by $\left\{e^{i n \lambda} e_{n} ; n \in \mathbb{N}\right\}$ and consider the pre-Hilbert bundle over $S$ with fibers $\left(H_{\lambda}\right)(\lambda \in S)$. Define the sections

$$
\lambda \in S \mapsto s_{\lambda}:=\sum_{n=1}^{\infty} e^{i n \lambda} e_{n} \in N^{*},
$$

where the limit in (6.4) is in the sense $\sigma\left(N^{*}, H_{0}\right)$. One can prove that the distributions $s_{\lambda}$ are in the domain of $\langle\cdot, \cdot\rangle_{c}$ and that $\left\langle s_{\lambda}, s_{\lambda}\right\rangle_{c}=1$. The set $\mathcal{H}_{2}(S)$ of all "weighted sections"

$$
\tilde{s}_{\varphi}:=\int_{S}^{\oplus} \varphi(\lambda) s_{\lambda} d \lambda \in \int_{S}^{\oplus} H_{\lambda} d \lambda ; \quad \varphi \in L^{2}(S, d \lambda)
$$

is a complex vector space on which we define the pre-scalar product

$$
\left\langle\tilde{s}_{\varphi}, \tilde{s}_{\psi}\right\rangle_{\mathcal{H}_{2}(S)}=\left\langle\int_{S}^{\oplus} \varphi(\lambda) s_{\lambda} d \lambda, \int_{S}^{\oplus} \psi(\lambda) s_{\lambda} d \lambda\right\rangle_{\mathcal{H}_{2}(S)}:=\int_{S}\left\langle\varphi(\lambda) s_{\lambda}, \psi(\lambda) s_{\lambda}\right\rangle_{c} d \lambda .
$$

Due to the identity

$$
\left\langle\tilde{s}_{\varphi}, \tilde{s}_{\psi}\right\rangle_{\mathcal{H}_{2}(S)}:=\int_{S} \bar{\varphi}(\lambda) \psi(\lambda)\left\langle s_{\lambda}, s_{\lambda}\right\rangle_{c} d \lambda=\langle\varphi, \psi\rangle_{L^{2}(S, d \lambda)}
$$

the correspondence

$$
\varphi \in L^{2}(S, d \lambda) \leftrightarrow \tilde{s}_{\varphi}=\int_{S}^{\oplus} \varphi(\lambda) s_{\lambda} d \lambda \in \mathcal{H}_{2}(S)
$$

is a unitary isomorphism between $\mathcal{H}_{2}(S)$ and $L^{2}(S, d \lambda)$.

Theorem 6.1. ${ }^{3}$ The linear map

$$
s: \varphi \in L^{2}(S, d \lambda) \rightarrow s_{\varphi}:=\int_{S} \varphi(\lambda) s_{\lambda} d \lambda \in N^{*}
$$

is well defined. Moreover, if we denote by $\mathcal{H}(S)$ the space of all distributions $s_{\varphi}, \varphi \in$ $L^{2}(S, d \lambda)$ and

$$
\hat{P}_{n]}\left(s_{\varphi}\right):=\int_{S} \varphi(\lambda) P_{n]}\left(s_{\lambda}\right) d \lambda,
$$

then the sesquilinear form

$$
\left\langle s_{\varphi}, s_{\psi}\right\rangle_{\mathcal{H}(S)}:=\lim _{n \rightarrow \infty} \frac{1}{n} \operatorname{Tr}\left(\hat{P}_{n]}\left|s_{\varphi}\right\rangle\left\langle s_{\psi}\right| \hat{P}_{n]}\right) ; \quad \varphi, \psi \in L^{2}(S, d \lambda)
$$

is well defined and satisfies

$$
\left\langle s_{\varphi}, s_{\psi}\right\rangle_{\mathcal{H}(S)}=\left\langle s_{\varphi}, s_{\psi}\right\rangle_{c}=\langle\varphi, \psi\rangle_{L^{2}(S, d \lambda)} .
$$


Definition 6.2. In the notations of the above theorem, the separable Hilbert space $\mathcal{H}(S)$ with scalar product $\langle\cdot, \cdot\rangle_{c}$ will be denoted $\mathcal{H}_{c}$ and called the Cesàro Hilbert space associated to $e=\left(e_{n}\right)_{n}$.

\subsection{The $\alpha$-extended Cesàro pre-Hilbert spaces}

Let $\mathcal{H}_{c, r}$ be the Cesàro Hilbert space constructed over the real standard triple

$$
E \subseteq H \subseteq E^{*}
$$

with respect to the orthonormal basis $e=\left(e_{n}\right)_{n} \subseteq E$ of $H$. We consider the complex Hilbert space $\mathcal{H}_{c}$ obtained by complexification of $\mathcal{H}_{c, r}$; i.e.

$$
\mathcal{H}_{c}=\mathcal{H}_{c, r}+i \mathcal{H}_{c, r}
$$

Lemma 6.1. The space (6.1) $\mathcal{H}_{c}$ coincides with the Cesàro Hilbert space constructed over the complex standard triple $N \subseteq K \subseteq N^{*}$, (cf. (6.1)).

Notice that the hermitian scalar product on $K$ is defined, for $\xi=\xi_{1}+i \xi_{2} \in K$, $\eta=\eta_{1}+i \eta_{2} \in K$, by:

$$
\begin{aligned}
\langle\xi, \eta\rangle_{K} & =\left\langle\xi_{1}+i \xi_{2}, \eta_{1}+i \eta_{2}\right\rangle_{K} \\
& =\left\langle\xi_{1}, \eta_{1}\right\rangle_{H}+\left\langle\xi_{2}, \eta_{2}\right\rangle_{H}-i\left\langle\xi_{2}, \eta_{1}\right\rangle_{H}+i\left\langle\xi_{1}, \eta_{2}\right\rangle_{H} .
\end{aligned}
$$

Moreover, the compatibility of the hermitian scalar product on $K$ and the duality $\left\langle N^{*}, N\right\rangle$ is given by:

$$
\langle z, \xi\rangle_{N^{*} \times N}=\langle\bar{z}, \xi\rangle_{K} ; \quad \forall z \in K, \quad \forall \xi \in N .
$$

Lemma 6.2. For $z \in \mathcal{H}_{c} \subseteq N^{*}$, we have, for any $n \in \mathbb{N}$

$$
\operatorname{Re}\left(\left\langle z, e_{n}\right\rangle_{N^{*} \times N}\right)=\left\langle\operatorname{Re} z, e_{n}\right\rangle_{E^{*} \times E} ; \quad \operatorname{Im}\left(\left\langle z, e_{n}\right\rangle_{N^{*} \times N}\right)=\left\langle\operatorname{Im} z, e_{n}\right\rangle_{E^{*} \times E} .
$$

Proof. Let $z \in \mathcal{H}_{c}$. By writing $z=\operatorname{Re} z+i \operatorname{Im} z$, with $\operatorname{Re} z$ and $\operatorname{Im} z$ elements of $\mathcal{H}_{c, r} \subseteq E^{*}$, we have

$$
\left\langle z, e_{n}\right\rangle_{N^{*} \times N}=\left\langle\operatorname{Re} z+i \operatorname{Im} z, e_{n}+i 0\right\rangle_{N^{*} \times N}=\left\langle\operatorname{Re} z, e_{n}\right\rangle_{E^{*} \times E}+i\left\langle\operatorname{Im} z, e_{n}\right\rangle_{E^{*} \times E}
$$

from which the identities (6.9) follow.

We recall the following lemma from Ref. 3 .

Lemma 6.3. In the notation (6.1), for each $\alpha>1 / 2$, we have the continuous embedding

$$
\mathcal{H}_{c} \subseteq N_{-\alpha}
$$

More precisely,

$$
|\xi|_{-\alpha}^{2} \leq C_{\alpha}\|\xi\|_{c}^{2} ; \quad \forall \xi \in \mathcal{H}_{c}
$$


where

$$
C_{\alpha}=\pi\left(\sum_{k=1}^{\infty} \frac{1}{(2 k+2)^{2 \alpha}}\right) .
$$

In the following, $\alpha$ is a fixed real number such that $\alpha>1 / 2$. Since

$$
e=\left(e_{n}\right)_{n} \subseteq E \equiv \bigcap_{p \geq 0} E_{p}
$$

one has, for any $n \in \mathbb{N}, e_{n} \in E_{\alpha}$. Moreover, Lemma 6.3 guarantees that the duality $\left\langle\mathcal{H}_{c}, N_{\alpha}\right\rangle$ is well defined by the pairing $\left\langle N^{*}, N\right\rangle$. On the other hand, any element of $N_{\alpha}$ has zero Cesàro-norm, therefore

$$
\mathcal{H}_{c} \cap N_{\alpha} \equiv\{0\}
$$

This allows one to introduce the direct sum of vector spaces

$$
\mathcal{H}_{\oplus}^{\alpha}:=\mathcal{H}_{c} \oplus N_{\alpha} \subseteq N^{*}
$$

On this vector space we define the following sesquilinear form: for $\xi, \eta \in \mathcal{H}_{c}$, $x, y \in N_{\alpha}$,

$$
\langle\xi \oplus x, \eta \oplus y\rangle_{\oplus, \alpha}:=C_{\alpha}\langle\xi, \eta\rangle_{\mathcal{H}_{c}}+\langle\xi, y\rangle_{\mathcal{H}_{c} \times N_{\alpha}}+\langle x, \eta\rangle_{N_{\alpha} \times \mathcal{H}_{c}}+\langle x, y\rangle_{N_{\alpha}}
$$

where $C_{\alpha}$ is given in Lemma 6.3.

Lemma 6.4. The sesquilinear form (6.13) is a pre-scalar product on $\mathcal{H}_{\oplus}^{\alpha}$.

Proof. For $\xi \in \mathcal{H}_{c}$ and $x \in N_{\alpha}$, one has

$$
\langle\xi \oplus x, \xi \oplus x\rangle_{\oplus, \alpha}=C_{\alpha}\|\xi\|_{c}^{2}+\|x\|_{\alpha}^{2}+2 \operatorname{Re}\left(\langle\xi, x\rangle_{\mathcal{H}_{c} \times N_{\alpha}}\right) .
$$

This gives

$$
\left|\langle\xi, x\rangle_{\mathcal{H}_{c} \times N_{\alpha}}\right| \leq|\xi|_{-\alpha}|x|_{\alpha} \leq \frac{1}{2}\left(|\xi|_{-\alpha}^{2}+|x|_{\alpha}^{2}\right) .
$$

Then, by (6.11), we obtain

$$
\left|\langle\xi, x\rangle_{\mathcal{H}_{c} \times N_{\alpha}}\right| \leq \frac{1}{2}\left(C_{\alpha}\|\xi\|_{c}^{2}+\|x\|_{\alpha}^{2}\right) .
$$

Therefore

$$
\left|2 \operatorname{Re}\langle\xi, x\rangle_{\mathcal{H}_{c} \times N_{\alpha}}\left\|\leq 2\left|\langle\xi, x\rangle_{\mathcal{H}_{c} \times N_{\alpha}}\right| \leq C_{\alpha}\right\| \xi\left\|_{c}^{2}+\right\| x \|_{\alpha}^{2}\right.
$$

which implies

$$
2 \operatorname{Re}\langle\xi, x\rangle_{\mathcal{H}_{c} \times N_{\alpha}}+C_{\alpha}\|\xi\|_{c}^{2}+\|x\|_{\alpha}^{2} \geq 0
$$

Definition 6.3. Let $\alpha>1 / 2$ be a fixed real number. By the $\alpha$-extended Ces̀aro pre-Hilbert space we mean the vector space (6.12) equipped with the pre-scalar product (6.13). 


\subsection{The associated quantum Lévy-Laplacian}

In the notations of the previous section, take $\mathcal{K}_{S}=\mathcal{H}_{\oplus}^{\alpha}$ and $T$ a real linear operator $T$ on $\mathcal{K}_{S}$. By definition, for any $z \in \mathcal{H}_{c}$, we have

$$
\begin{aligned}
\|z\|_{\oplus, \alpha}^{2} & =C_{\alpha}\|z\|_{c}^{2}=C_{\alpha}\left(\|\operatorname{Re} z\|_{c}^{2}+\|\operatorname{Im} z\|_{c}^{2}\right) \\
& =C_{\alpha} \lim _{n \rightarrow+\infty} \frac{1}{n} \sum_{k=1}^{n}\left(\left\langle e_{k}, \operatorname{Re} z\right\rangle_{E \times E^{*}}^{2}+\left\langle e_{k}, \operatorname{Im} z\right\rangle_{E \times E^{*}}^{2}\right) .
\end{aligned}
$$

On the other hand, since $\mathcal{H}_{\oplus}^{\alpha}$ contains both $\mathcal{H}_{c}$ and the vectors $e_{k}, k \in \mathbb{N}$, we can use the identities (4.13), (4.14), so that for any $k \in \mathbb{N}, z \in \mathcal{H}_{c}$

$$
\left[p_{0}\left(e_{k}\right), W_{0}(z)\right]=\sqrt{2} \operatorname{Re}\left(\left\langle e_{k}, z\right\rangle_{N \times N^{*}}\right) W_{0}(z)=\sqrt{2}\left\langle e_{k}, \operatorname{Re} z\right\rangle_{E \times E^{*}} W_{0}(z)
$$

and

$$
\left[q_{0}\left(e_{k}\right), W_{0}(z)\right]=-\sqrt{2} \operatorname{Im}\left(\left\langle e_{k}, z\right\rangle_{N \times N^{*}}\right) W_{0}(z)=-\sqrt{2}\left\langle e_{k}, \operatorname{Im} z\right\rangle_{E \times E^{*}} W_{0}(z) .
$$

Hence

$$
\begin{aligned}
& {\left[p_{0}\left(e_{k}\right),\left[p_{0}\left(e_{k}\right), W_{0}(z)\right]\right]=2\left\langle e_{k}, \operatorname{Re} z\right\rangle_{E \times E^{*}}^{2} W_{0}(z)} \\
& {\left[q_{0}\left(e_{k}\right),\left[q_{0}\left(e_{k}\right), W_{0}(z)\right]\right]=2\left\langle e_{k}, \operatorname{Im} z\right\rangle_{E \times E^{*}}^{2} W_{0}(z) .}
\end{aligned}
$$

It follows that, for any $z \in \mathcal{H}_{c}$,

$$
\begin{aligned}
& \frac{1}{2}\|z\|_{\oplus, \alpha}^{2} W_{0}(z) \\
& \quad=\frac{C_{\alpha}}{4} \lim _{n \rightarrow+\infty} \frac{1}{n} \sum_{k=1}^{n}\left(\left[p_{0}\left(e_{k}\right),\left[p_{0}\left(e_{k}\right), W_{0}(z)\right]\right]+\left[q_{0}\left(e_{k}\right),\left[q_{0}\left(e_{k}\right), W_{0}(z)\right]\right]\right) .
\end{aligned}
$$

Definition 6.4. The quantum Lévy-Laplacian associated to $\mathcal{H}_{c, r}$ is the operator, denoted $\Delta_{L}^{Q}$, defined, with respect to the basis $e=\left(e_{k}\right)_{k}$ of $H$, by

$$
\Delta_{L}^{Q}:=\frac{C_{\alpha}}{4} \lim _{n \rightarrow+\infty} \frac{1}{n} \sum_{k=1}^{n}\left(\left[p_{0}\left(e_{k}\right),\left[p_{0}\left(e_{k}\right), \cdot\right]\right]+\left[q_{0}\left(e_{k}\right),\left[q_{0}\left(e_{k}\right), \cdot\right]\right]\right),
$$

where $H$ is the middle Hilbert space of the real standard triple on which the real Cesáro Hilbert space $\mathcal{H}_{c, r}$ is constructed.

Remark 6.1. From the previous discussion we have, for any $z \in \mathcal{H}_{c}$,

$$
\Delta_{L}^{Q}\left(W_{0}(z)\right):=\frac{1}{2}\|z\|_{\oplus, \alpha}^{2} W_{0}(z)=\frac{C_{\alpha}}{2}\|z\|_{c}^{2} W_{0}(z)=C_{\alpha} \Delta^{Q}\left(W_{0}(z)\right) .
$$

This means that the quantum Lévy-Laplacian $\Delta_{L}^{Q}$ is proportional to the quantum Volterra Laplacian $\Delta_{V}^{Q} \equiv \Delta^{Q}$. Moreover, for any $z \in \mathcal{H}_{c}$, the Weyl operator $W_{0}(z)$ is an eigenvector for $\Delta_{L}^{Q}$ associated to the eigenvalue $\frac{C_{\alpha}}{2}\|z\| c^{2}$. So that the spectrum of $\Delta_{L}^{Q}$ contains the interval $[0, \infty)$. In fact, this is the quantum analogue of the classical picture with Lévy-Laplacian $\Delta_{L}$ and distributions $\delta_{z}, z \in \mathcal{H}_{c}$, (see Ref. 6). 


\section{Acknowledgment}

This work was supported by Università degli Studi di Roma "Tor Vergata", grantin-aid for scientific research No. 1490-04.

\section{References}

1. L. Accardi, A note on Meyer's note, in Quantum Probability and Applications III, Oberwolfach 1987 Lecture Notes in Mathematics, Vol. 1303, ed. L. Accardi, (SpringerVerlag, 1988), pp. 1-5.

2. L. Accardi, On the Quantum Feynman-Kac Formula, Rendiconti del Seminario Mathematico e Fisico di Milano, Vol. XLVIII (1978).

3. L. Accardi, A. Barhoumi and H. Ouerdiane, Cesàro Hilbert Space and the LévyLaplacian, Semin. Congr. Math., France (2005), to appear.

4. L. Accardi, Y. G. Lu and I. Volovich, Nonlinear extensions of classical and quantum stochastic calculus and essentially infinite dimentional analysis, in Probability Towards 2000, Springer Lecture Notes in Statistics, Vol. 128, eds. L. Accardi and C. Heyed (Springer-Verlag, 1998), pp. 1-33.

5. L. Accardi and O. G. Smolyanov, On Laplacians and Traces, Rend. Sem. Math. Bari, October 1993, Volterra preprint.

6. L. Accardi, H. Ouerdiane and O. G. Smolyanov, Lévy-Laplacian acting on operators, Russ. J. Math. Phys. 10 (2003) 359-380.

7. L. Accardi and H. Ouerdiane, Semi-groupe Associé à L'opérateur de Laplace-Lévy, Volterra preprint No. 509 April (2002).

8. L. Accardi, W. Ayed and H. Ouerdiane, White noise approach to stochastic integration, preprint 2004.

9. W. Arveson, Dynamical invariants for noncommutative flows, Operator Algebras and Quantum Field Theory (Rome, 1996) (Internat. Press, Cambridge, MA, 1997), pp. $476-514$.

10. W. Arveson, The heat flow of the CCR algebra, preprint 2000, available at http://www.math.berkely.edu/ arveson.

11. W. Arveson, The domain algebra of a CP-semigroup, Pac. J. Math. 203 (2002).

12. W. Arveson, Noncommutative Dynamics and E-Semigroups, Springer Monographs in Mathematics Series (Springer, 2003).

13. A. Barchielli, Input and output channels in quantum stochastic differential equation, Quantum Probability and Applications III, Lecture Notes in Mathematics, Vol. 1303, (Springer-Verlag, 1988).

14. F. Fagnola, Quantum Markov semi-groups and quantum Markov flows, Tesi di Perfizionamento. Scola Normale Superiore di Pisa, Pisa (1998).

15. R. Gannoun, R. Hachaichi, H. Ouerdiane and A. Rezgi, Un Théorème de Dualité Entre Espace de Fonctions Holomorphes à Croissance Exponentielle, J. Funct. Anal. 171 (2000) 1-14.

16. I. M. Gel'fand and N. Ya. Vilenkin, Generalized Functions (Academic Press, 1964), Vol. 4.

17. T. Hida, Brownian Motion (Springer-Verlag, 1980).

18. T. Hida, A role of the Lévy-Laplacian in the causal calculus of generalized white noise functionals, Stochastic Process, A Festschrift in Honour of G. Kallianpur, eds. S. Cambanis et al. (Springer-Verlag, 1992).

19. T. Hida, H.-H. Kuo, J. Potthoff and L. Streit, White Noise: An Infinite Dimensional Calculus (Kluwer, 1993). 
20. J. L. Journé, Structures des cocycles Markoviens sur l'espace de Fock, Probab. Th. Rel. Fields 75 (1987) 291-316.

21. H.-H. Kuo, White Noise Distrubition Theory (CRC Press, 1996).

22. H.-H. Kuo, On Laplacian Operators of Generalized Brownian functionals, Lecture notes in Math., Vol. 1203, (Springer-Verlag, 1986), pp. 119-128.

23. H.-H. Kuo, Recent progress on the white noise approach to the Lévy-Laplacian, LSU Mathematics Electronic Preprint Series, 2003.

24. H.-H. Kuo, N. Obata and K. Saitô, Diagonalization of the Lévy-Laplacian and related stable Processes, Inf. Dim. Anal. Quantum Probab. Rel. Topics 5 (2002) 317-331.

25. G. Lindblad, Brownian motion of a quantum Harmonic oscillator, Rep. Math. Phys. 10 (1976) 393-406.

26. P. Lévy, Leçons d'analyse Fonctionelles (Gauthier-Villars, 1922).

27. P. A. Meyer, A note on shifts and cocycles, Quantum Probability and Applications III, Lecture Notes in Mathematics, Vol. 1303, eds. L. Accardi and W. Waldenfelds (Springer-Verlag, 1988), . 361-374.

28. N. Obata, White Noise Calculus and Fock Spaces, Lecture Notes in Mathematics, Vol. 1577 (Springer-Verlag, 1994).

29. N. Obata, A characterization of the Lévy-Laplacian in termes of infinite dimensional rotation groups, Nagoya Math. J. 118 (1990) 111-132.

30. N. Obata and H. Ouerdiane, Heat equation associated with Lévy Laplacian, preprint (2001) in Proc. Int. Conf. on Stochastic Analysis and Applications, eds. S. Albeverio, A. B. de Monvel and H. Ouerdiane (Kluwer, 2004), pp. 53-68.

31. K. R. Parthasarathy, An Introduction to Quantum Stochastic Calculus (Birkhäuser, 1992).

32. K. Saitô, A $\left(C_{0}\right)$-group generated by the Lévy-Laplacian, Inf. Dim. Anal. Quantum Probab. Rel. Topics 1 (1998) 425-437. 